Revue des patrimoines

\title{
Les collections de modèles anatomiques équins de Louis Auzoux, une collection à constituer
}

\section{Christophe Degueurce}

\section{(2) OpenEdition}

\section{Journals}

Édition électronique

URL : http://journals.openedition.org/insitu/11950

DOI : $10.4000 /$ insitu. 11950

ISSN : 1630-7305

\section{Éditeur}

Ministère de la culture

Référence électronique

Christophe Degueurce, "Les collections de modèles anatomiques équins de Louis Auzoux, une collection à constituer », In Situ [En ligne], 27 | 2015, mis en ligne le 02 novembre 2015, consulté le 19 avril 2019. URL : http://journals.openedition.org/insitu/11950; DOI : 10.4000/insitu.11950

Ce document a été généré automatiquement le 19 avril 2019

\section{(c) $($ i) $(9)$}

In Situ Revues des patrimoines est mis à disposition selon les termes de la licence Creative Commons Attribution - Pas d'Utilisation Commerciale - Pas de Modification 4.0 International. 


\title{
Les collections de modèles anatomiques équins de Louis Auzoux, une collection à constituer
}

\author{
Christophe Degueurce
}

L'auteur remercie tout particulièrement M. François Dubosc, responsable du musée du Neubourg, et M. Bernard Barral, qui administra les établissements du docteur Auzoux pendant une grande partie du XXe siècle. Ses pensées vont également à Barbara Dumont, Manu Giaccone, Caroline Marchal, Pauline Morlot, Elisabeth Nijhof-Asser, Jim Poncelet, Ségolène Walle, qui ont restauréle cheval du musée Fragonard, et à Pfizer Santé animale qui a financé ce sauvetage.

1 L'anatomiste cherche dans la chair une vérité d'organisation qui, dès lors qu'elle est révélée, se délite et disparaît sous l'effet de la putréfaction. Les hommes de l'art ont donc systématiquement cherché à lutter contre ce processus naturel qui transforme la précise dissection en un amas putride qui ne peut que retourner à sa destinée primitive.

2 Tous les anatomistes ont cherché à stopper le terrible délitement pour garder le fruit de leur habile ouvrage. L'art de la préparation anatomique connut un tournant au XVIII ${ }^{\mathrm{e}}$ siècle, à une époque où l'anatomie macroscopique de l'homme était connue et où l'enseignement prit le pas sur les recherches. Montrer les formations anatomiques et conserver les traces du passage du prosecteur furent des objectifs pédagogiques autant qu'institutionnels, les larges collections des établissements de la fin du Siècle des Lumières témoignant de leur aura. Deux techniques étaient alors en vigueur; la principale était qualifiée d'« anatomie naturelle » et passait par la conservation des corps véritables, entiers ou fragmentés, desséchés ou conservés dans des bains. La première catégorie impliquait le recours à la momification, c'est-à-dire la dessiccation du corps, au retrait des fluides des cellules qui permettent normalement aux agents biologiques de se développer et de dégrader les tissus. Nombreux furent ceux qui excellèrent dans ce domaine et, si l'histoire a essentiellement retenu le nom d'Honoré Fragonard (1732-1799), des préparations de ce type peuplaient les cabinets anatomiques des facultés de médecine de l'Europe entière comme les collections privées de quelques riches "curieux $\|^{1}$. Ces corps desséchés étaient d'utilisation complexe car la rétraction des formations 
anatomiques transformait les muscles rebondis en lanières reflétant peu leur conformation initiale. L'alternative à cette anatomie transformée était de plonger les organes dans des bains de solutions conservatrices. La chose convenait parfaitement à des portions de corps, permettant la conservation et même la dissection ultérieure, mais était complexe pour des spécimens entiers.

Les anatomistes firent donc appel à des intermédiaires, des artistes, qui étaient capables de recréer des simulacres d'anatomie. Chaque matériau eut ainsi ses indications, ses limites et fut exploité pour mimer telle ou telle partie du corps. La cire teintée, délicate et fragile, donnait sa translucidité et sa brillance à la restitution des parties tant internes qu'externes. Le plâtre, plus grossier, moulait sommairement le corps et ses parties, restant blanc pour des épreuves de travail ou se couvrant d'à-plats colorés lorsqu'il était destiné à être conservé. Même le verre connut son heure de gloire dans le domaine de l'anatomie animale, formant les tissus transparents des invertébrés marins ${ }^{2}$. Mais il est un matériau qui connut au XIX ${ }^{e}$ siècle un succès planétaire, une notoriété qui contraste avec l'oubli dans lequel il se trouve aujourd'hui. Cette technique a laissé un nom, celui de Louis Auzoux (1797-1880), un médecin qui développa une entreprise florissante qui devait produire des modèles en papier mâché, démontables à l'envi et mimant la dissection, exportés vers les cinq continents et qui peuplent aujourd'hui encore de nombreuses collections.

\section{Louis Auzoux et les modèles anatomiques en papier mâché}

Ce n'est pas le lieu ici d'exposer en détail la biographie de cet inventeur de génie et le lecteur pourra se reporter à d'autres documents plus élaborés ${ }^{3}$ mais quelques éléments ponctuels sont cependant utiles. Louis naquit en 1797 dans une famille de cultivateurs aisés du village de Saint-Aubin-d'Écrosville, près d'Évreux. Il suivit des études brillantes, terminant par des études de médecine à Paris qui lui firent rencontrer les personnalités du monde médical parisien du début du XIX ${ }^{e}$ siècle. Quelles furent les circonstances exactes qui le poussèrent à se lancer dans cette aventure? Difficile de le dire tant les relations de ses débuts sont enthousiastes et servent le souvenir de celui qui allait être une personnalité du siècle de l'industrie. Ce qui est certain, c'est que Louis Auzoux engagea ses recherches très tôt, durant ses études, et produisit des efforts considérables et coûteux pour mettre en place sa méthode. Peu de doute qu'il ne se soit inspiré des travaux d'un autre Normand, plus âgé, Jean-François Ameline, professeur d'anatomie à Caen qui connaissait alors une gloire modeste pour avoir eu l'idée de créer des mannequins d'un genre nouveau, où les formations anatomiques étaient mimées en carton et accrochées à un squelette véritable. Le corps ainsi assemblé pouvait être démonté à la manière d'une dissection révélant, couche après couche, les éléments de plus en plus profonds, le passage d'un nerf ou d'un vaisseau entre des muscles, les rapports d'un viscère dans la cage thoracique ou l'abdomen. Le résultat était spectaculaire au premier abord, moins au second car, si l'idée était géniale, le rendu de bon nombre d'éléments était insuffisant. Il présenta à partir de 1816 des pièces à la société de médecine de Caen ${ }^{4}$ avant de gagner les cercles savants de Paris qui, de 1819 à 1821 , louèrent tous son travail. Le jeune Auzoux, alors Parisien, eut connaissance de cette méthode et fit même le déplacement à Caen pour visiter l'atelier de son aîné. 
5 En septembre 1822, il présentait à l'Académie royale de médecine un "membre abdominal $\Perp^{5}$, selon un procédé voisin de celui d'Ameline, également sur des os naturels et concurrençait de fait son créateur. La présentation d'une nouvelle pièce, une tête, un cou et la partie supérieure du tronc à la Société médicale d'émulation ${ }^{6}$ devait attirer sur lui l'attention du gouvernement, qui lui commanda un mannequin complet, pièce qu'il livra en 1825. Elle comportait une innovation majeure : le squelette était artificiel, ce qui facilitait sa reproduction. Ainsi s'initiait une carrière fructueuse qui allait voir la confection de plusieurs centaines de modèles artificiels, pouvant tous être produits en grand nombre, de façon quasi industrielle, à un coût abordable assurant leur diffusion. Ce modèle fut remanié et fournit le grand modèle de 1830, qui devait être produit jusque dans les années 1970. Auzoux produisit ensuite une femme dans la position de la Vénus de Médicis, puis créa de nombreux modèles d'organes isolés ou assemblés au sein de régions du corps. En 1834, ces pièces d'anatomie artificielle prenaient le qualificatif de " clastique », d'une racine grecque signifiant « rompre, séparer »"

6 Mais Auzoux n'en resta pas aux humains et, en naturaliste éclectique, se passionna pour l'anatomie animale. Fidèle au mouvement zoologique s'appuyant sur la classification et l'anatomie comparée, il réalisa des animaux «types », c'est-à-dire représentant selon lui une branche de cette classification. C'est ainsi que furent produits le dindon, « comme type des volatiles ", un squale " comme type des poissons cartilagineux » et le hanneton " comme type des insectes à l'état adulte ». La série continua avec la perche de mer, type des poissons, la sangsue, comme type des vers annélides, ou encore le boa constrictor, comme type des reptiles.

7 Quelques animaux furent plus particulièrement détaillés, ceci du fait de leur importance dans l'économie humaine. Ce fut le cas de l'abeille, représentée sous toutes ses formes dans une mise en scène époustouflante, ou du ver à soie dont il créa les modèles de la chenille et des adultes des deux sexes. Le cheval surtout devait tenir une place particulière dans son œuvre. Il faut dire que l'animal était au cœur de tous les enjeux : animal de travail, il était le moteur diesel de l'économie humaine; cheval de luxe, il servait à la distinction sociale du propriétaire; cheval militaire, il était un enjeu stratégique majeur. Pas étonnant donc que le premier modèle équin fut créé parmi les premiers qui suivirent les modèles humains.

\section{La création du premier mannequin de cheval}

8 Louis Auzoux a dû engager très tôt la conception de cette œuvre monumentale. La première relation de sa démarche apparait dans ses archives en 1842, dans un échange de courrier qu'il a avec un jeune membre de sa famille, alors élève officier à l'École de cavalerie de Saumur. Ce dernier écrit à son parent pour lui signaler que son maître d'équitation, M. de Saint-Ange ${ }^{8}$, verrait un grand avantage pédagogique à bénéficier d'un mannequin équin bâti sur le modèle de l'homme dont l'École de cavalerie usait. Il se plaignait des pièces anatomiques desséchées et goudronnées qui ne rendaient qu'un aperçu très sommaire de l'anatomie de cet animal'.

Louis Auzoux lui répondait le 2 avril 1842, décrivant par le menu le fameux cheval, visiblement terminé; il exposait les difficultés terribles qu'il avait endurées, ceci notamment du fait de la rareté de l'iconographie anatomique dans cette espèce, et imaginait déjà une collaboration avec l'écuyer pour décliner le modèle en versions 
simplifiées et moins onéreuses, plus adaptées par exemple aux usages des militaires et à leur diffusion à large échelle. Surtout, il révélait que le projet était ancien, contemporain même de la toute première commande d'un homme complet par le gouvernement :

Les réductions et soustractions que j'ai faites pour l'homme pourront être faites pour le cheval. En 1825, le gouvernement d'alors m'avait proposé d'exécuter ce travail qui me donnait l'assurance d'en faire placer dans les écoles vétérinaires, dans chaque régiment de cavalerie, dans les cours d'hippiatrique, d'hygiène [...] L'homme m'avait fait ajourner ce projet, depuis plusieurs gouvernements étrangers m'ayant exprimé la même intention, je me suis attelé au cheval et pour la fin du mois je serai en mesure d'en montrer un premier exemplaire (fig. 1).

Figure 1

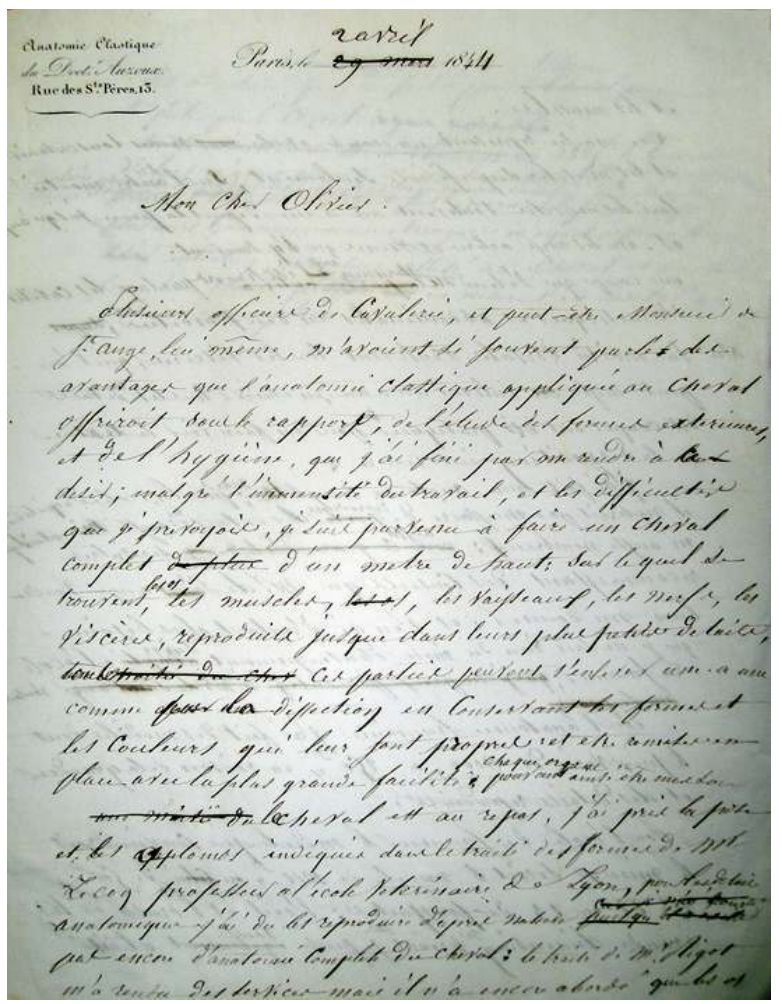

Lettre de Louis Auzoux à son jeune parent, élève officier à l'École royale de cavalerie de Saumur, datée du 2 avril 1844

Repro. Degueurce, Christophe. (c) Archives nationales, juin 2012.

Auzoux ne mentait pas; le processus en était à sa conclusion puisqu'il soumettait son mannequin à l'Académie royale de médecine en avril $1844^{10}$. Le dossier consacré à ce modèle, conservé aux Archives nationales dans le fonds Montaudon ${ }^{11}$, un des successeurs d'Auzoux, montre ce que ce pionnier avait à sa disposition. Tout juste trouve-t-on une planche remaniée d'après celles du fameux Cours d'hippiatrique que Philippe-Étienne Lafosse publia en $1772^{12}$ (fig. 2). Auzoux avait dû réaliser une étude anatomique tout à fait originale, basculant de celle de l'homme, qu'il connaissait, à celle du cheval, que les enseignants des écoles vétérinaires faisaient progresser :

Le cheval est au repos, j'ai pris la pose et les aplombs indiqués dans le traité des formations ${ }^{13}$ de $\mathrm{Mr}$ Lecoq, professeur à l'école vétérinaire de Lyon, pour les détails anatomiques, j'ai dû les reproduire d'après nature car il n'existe pas encore d'anatomie complète du cheval : le traité de Mr Rigot m'a rendu des services mais il n'a encore abordé que les os et les muscles ${ }^{14}$. 
Figure 2

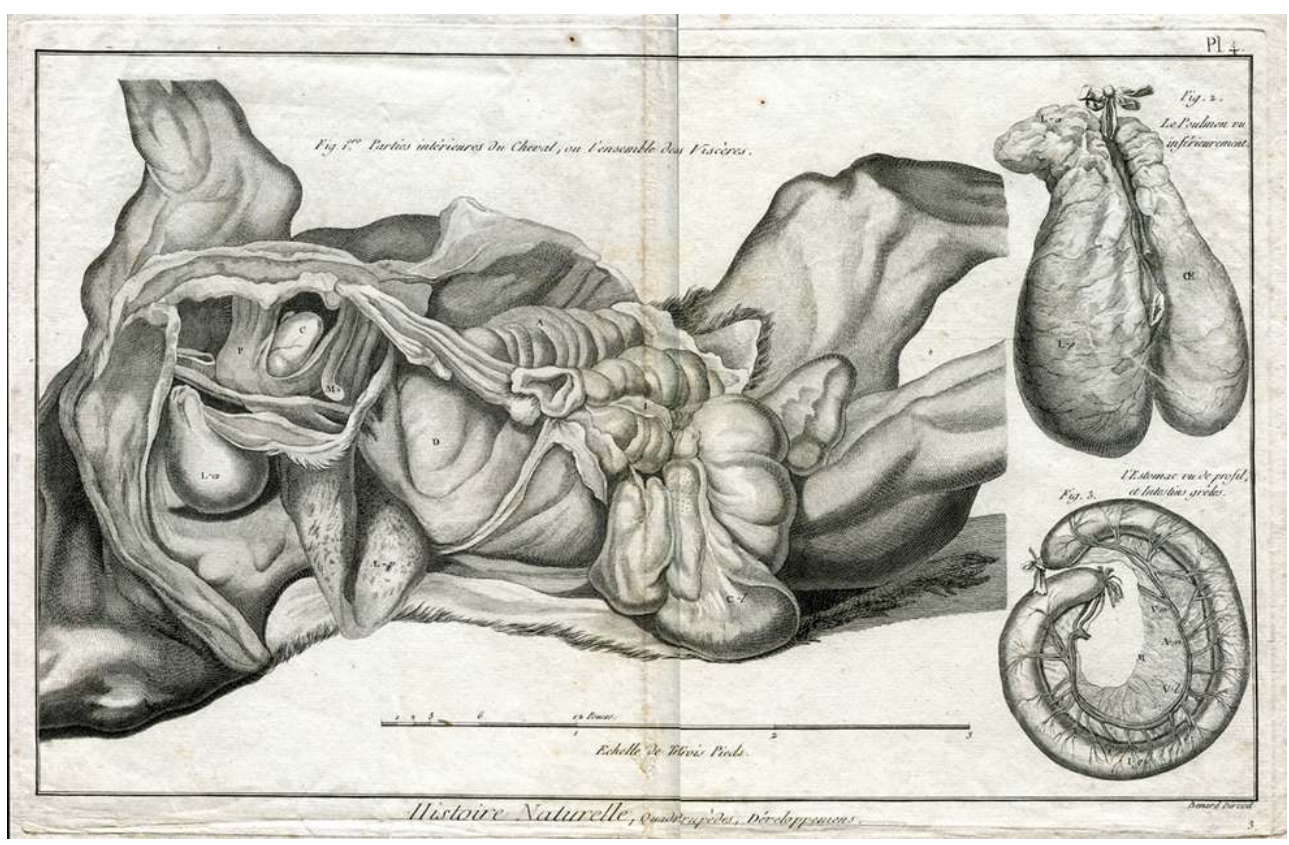

Planche rassemblant trois gravures issues du Cours d'Hippiatrique de Philippe-Étienne Lafosse (1772). Repro. Degueurce, Christophe. (c) Archives nationales, juin 2012

Il soumit du reste sa pièce au jugement de ces professionnels : le dossier d'archives présente plusieurs notes manuscrites prises alors qu'un de ces enseignants vétérinaires examinait son spécimen, et de nombreux billets de correspondance convenant de rendezvous pour ces évaluations. Du reste, Auzoux ne cachera pas les apports dont il avait bénéficié :

«M'occupant du cheval, j'ai dû nécessairement faire de nombreux emprunts aux écoles vétérinaires, soit pour la confection du cheval clastique, soit pour les altérations que présentent certains organes ${ }^{15}$. "

Il nommait dans le même ouvrage ces contributeurs dans ses Leçons élémentaires d'anatomie et de physiologie humaine et comparée en remerciant Félix Lecoq, alors directeur de l'École de Lyon, Eugène Renault ${ }^{16}$, directeur de celle d'Alfort, et Armand Goubaux ${ }^{17}$, professeur d'anatomie de ladite école. Il oubliait Félix Rigot, mort depuis onze ans mais qui avait aidé Auzoux de ses conseils ${ }^{18}$ (fig. 3). 
Figure 3

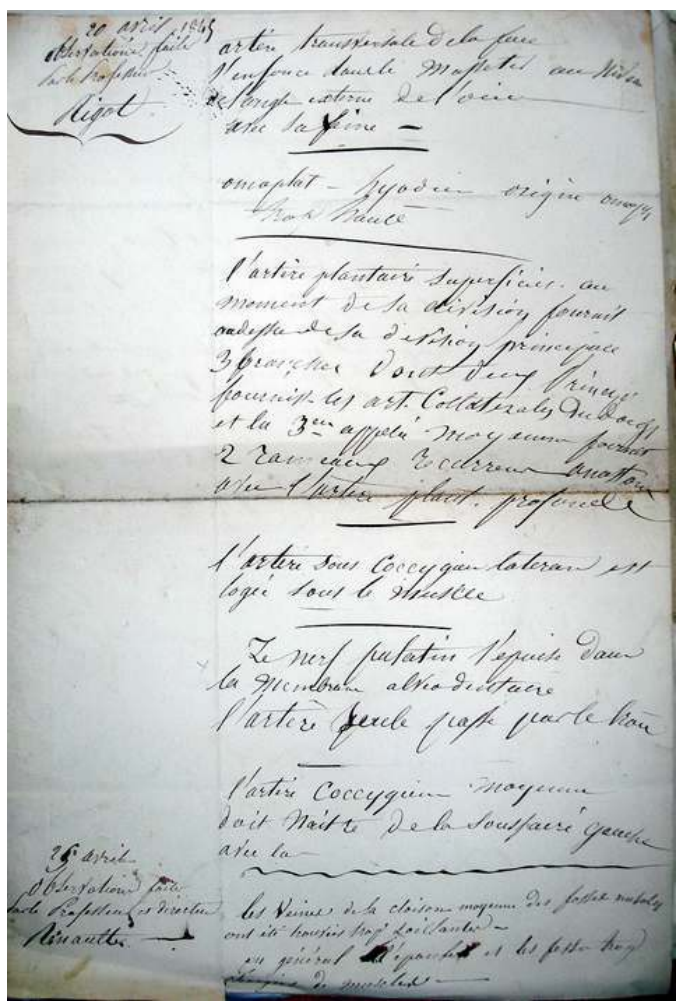

Billet manuscrit sur lequel sont consignées les remarques émises par les professeurs Rigot et Renault sur le cheval clastique (20 et 25 avril 1845).

Repro. Degueurce, Christophe. (c) Archives nationales, juin 2012.

Dans le domaine de l'agriculture et de l'élevage, Auzoux bénéficiait de l'amitié d'Antoine Richard, dit «Richard du Cantal», un personnage fascinant, tout à la fois vétérinaire, médecin, agriculteur, agronome, un temps enseignant à l'École de Grignon, enseignant puis directeur de l'École des haras, avant de suivre une carrière politique dans le même camp que celui d'Auzoux, celui des républicains ${ }^{19}$. Si Auzoux le cite plusieurs fois dans ce même ouvrage, c'est que Richard fut un scientifique de premier ordre, créant avec Geoffroy Saint- Hilaire la Société zoologique d'acclimatation, et réalisa des conférences de vulgarisation similaires à celles qu'Auzoux dispensait tous les dimanches dans son établissement parisien. Les deux hommes étaient faits pour s'entendre. Il publia des ouvrages de grande importance sur l'amélioration du cheval, comme De la Conformation du Cheval selon les lois de la Physiologie et de la Mécanique ${ }^{20}$ en 1847, réédité plusieurs fois et dont le titre évolua en 1857 pour devenir Étude du Cheval de service et de guerre suivant les principes élémentaires des Sciences naturelles ${ }^{21}$. Richard comme Lecoq devinrent des amis proches d'Auzoux; la correspondance qu'ils entretinrent les montre se rendant les uns chez les autres.

Le dernier compagnon, intervenant plus tardivement que les vétérinaires, fut FrançoisMaxime Jacquemin, la branche militaire en somme de la compagnie ${ }^{22}$. On peut affirmer qu'il voua un véritable culte à Auzoux, s'adressant à lui toujours d'une manière très révérencieuse. L'homme était passionné par l'hippologie et croyait dans l'instruction. Il faut dire qu'il avait dû, comme les militaires de sa génération - il avait fait les dernières campagnes napoléoniennes - être profondément marqué par les déboires de la Grande 
Armée, plusieurs fois meurtrie par des anomalies de gestion des effectifs équins qui auraient été facilement évitées si les soldats avaient été un tant soit peu formés au maintien de leurs chevaux. Il avait publié plusieurs ouvrages ayant trait à l'anatomie du cheval et à l'hippiatrique; il avait de fait tenté de former les jeunes recrues, faisant des régiments de petites écoles vétérinaires ${ }^{23}$. Jacquemin entretint avec Auzoux une correspondance très nourrie, l'aidant notamment au début des années 1850 dans la conception de l'anatomie du doigt du cheval, du sabot selon Bracy Clarck ou encore des pièces pathologiques, tares dures, molles et membres associés ${ }^{24}$.

\section{Les différents modèles de chevaux}

Auzoux avait produit un spécimen exceptionnel ${ }^{25}$, parfaitement décrit par les rapporteurs qui découvrirent, lors de la séance de l'Académie royale de médecine d'avril 1844, un équidé de $1,10 \mathrm{~m}$ de hauteur dont, comme les écorchés humains, la partie droite présentait les formations superficielles tandis que la moitié gauche pouvait être démontée pour faire apparaître les plans profonds. L'ouverture du cheval se faisait après le retrait des membres, puis la moitié dorsale du tronc, avec l'encolure et la tête, était levée, emportant avec elle l'ensemble des viscères (fig. 4, fig.5). Les rapporteurs notèrent quelques imperfections - le mannequin était «trop large de poitrine », avait les membres «trop volumineux », les muscles des régions postérieures « trop massifs », était disharmonieux en somme - mais louèrent la démarche et le résultat. Auzoux avait gagné son pari. Avec ce modèle, il disposait d'un type de mammifère, mais pas n'importe lequel, le cheval, l'animal stratégique par excellence ${ }^{26}$.

Figure 4

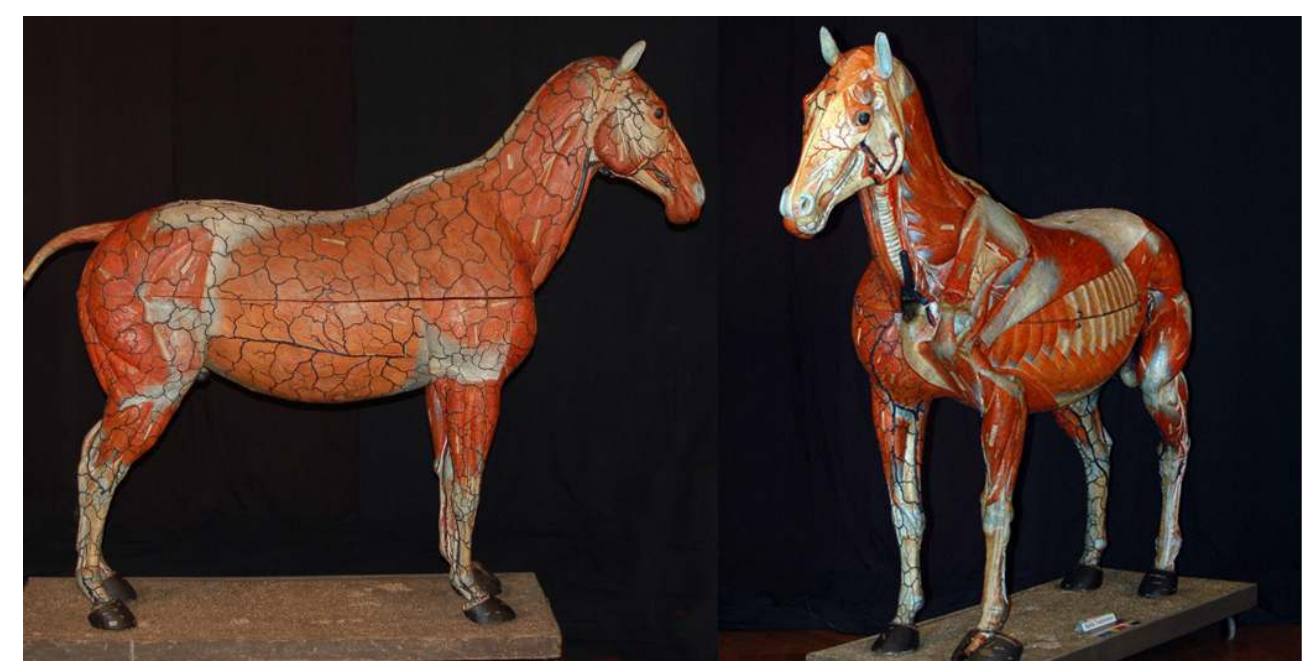

(a) Face droite du cheval, montrant les vaisseaux et muscles superficiels ; on voit nettement le plan de clivage entre les parties dorsale et ventrale du tronc permettant l'ouverture du cheval. Phot. Degueurce, Christophe. ( École nationale vétérinaire de Maisons-Alfort, mai 2012. (b) Vue crâniolatérale gauche du cheval, montrant les vaisseaux et muscles profonds ; on voit nettement le plan de clivage entre les parties dorsale et ventrale du tronc permettant l'ouverture du cheval.

Phot. Degueurce, Christophe. ( ) Musée de l'École nationale vétérinaire de Maisons-Alfort, mai 2012. 
Figure 5

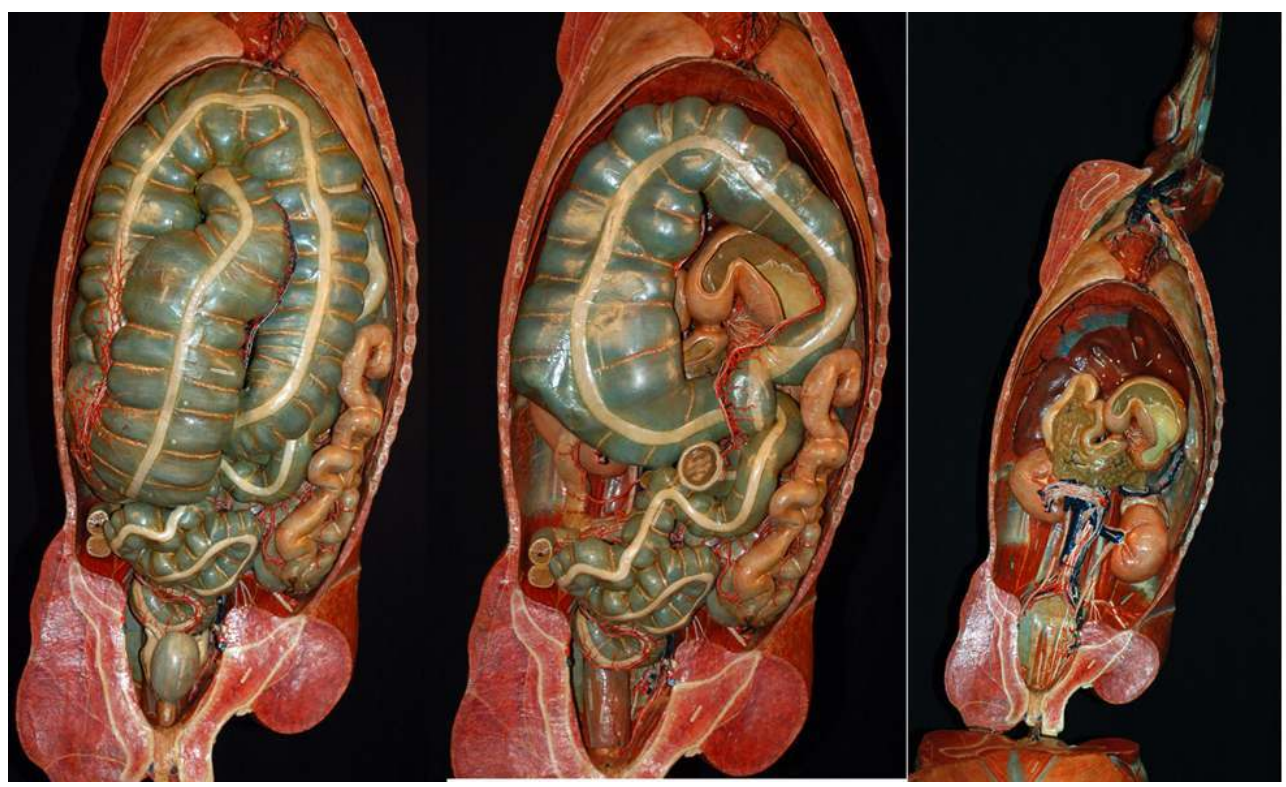

Vue de l'intérieur du tronc après ouverture. Plan 1 : Tous les organes sont en place et on voit parfaitement les replis du côlon ascendant et le caecum. Plan 2 : La partie ventrale du côlon ascendant et le caecum ont été ôtés, laissant apparaître la partie dorsale du côlon et les organes postdiaphragmatiques. Plan 3 : Le côlon ascendant et le caecum ont été ôtés, laissant apparaître les organes post-diaphragmatiques et les reins.

Phot. Degueurce, Christophe. (c) Musée de l'École nationale vétérinaire de Maisons-Alfort, mai 2012.

16 Il commercialisait ce modèle en 1845 , en lui joignant un livret, baptisé «Tableau synoptique $\aleph^{27}$ (fig. 6) dans lequel étaient référencées toutes les parties pouvant être détachées, indiquant comment elles devaient l'être et, pour chaque fragment, citant les noms des innombrables formations anatomiques qui étaient légendées. Ce cheval était dit complet car, sur le côté gauche, tous ses muscles se détachaient un à un. Il comprenait 127 pièces détachables et 3635 détails anatomiques ${ }^{28}$, bien plus que ce qu'un vétérinaire connaissait. Le prix de ce cheval était faramineux, 4000 francs, plus cher même que l'homme complet qui coûtait 3000 francs. Des modèles de ce cheval complet se trouvent dans les collections des Écoles nationales vétérinaires de Lyon et de Toulouse, tous deux sont datés de 1851. 
Figure 6

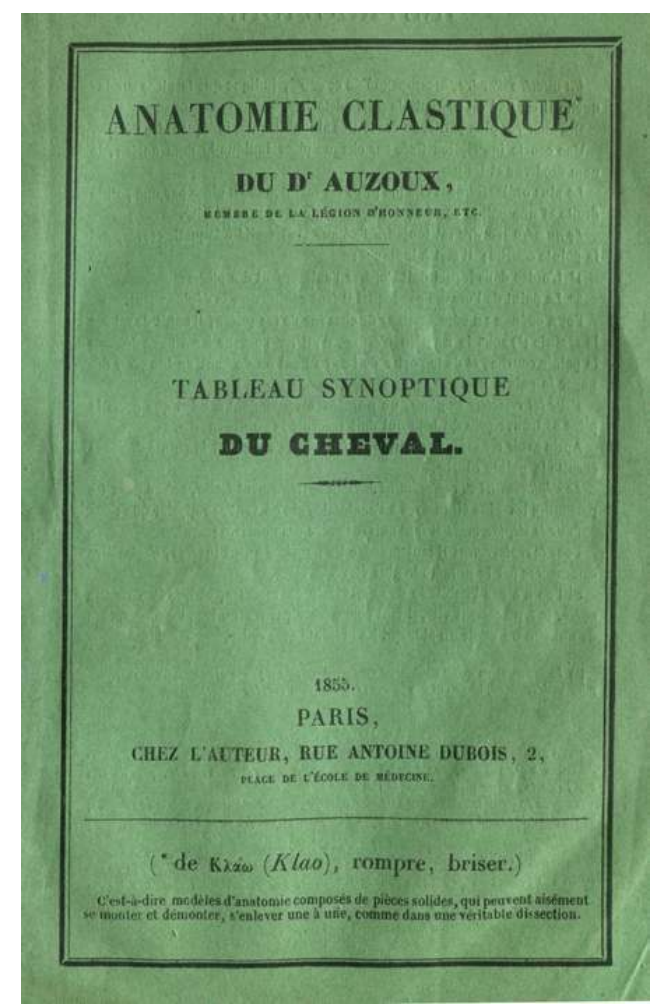

Couverture du Tableau synoptique du cheval clastique.

Repro. Degueurce, Christophe. (c) Degueurce, Christophe, juin 2012.

Répondant à la demande de M. de Saint-Ange et de plusieurs officiers de cavalerie, il commercialisa en même temps un modèle dit «cheval incomplet » destiné aux écoles de cavalerie, aux régiments de cavalerie et d'artillerie, et aux haras. Ce second spécimen était similaire au premier, de même taille, présentait les mêmes viscères; les muscles n'étaient pas détachables et un côté présentait les structures profondes tandis que l'autre était dévolu aux éléments superficiels. Il était formé de 19 pièces et présentait 1985 détails anatomiques ; il était accompagné du même tableau synoptique, simplement les numéros correspondant aux détails anatomiques absents n'étaient pas présents sur le cheval ${ }^{29}$. On peut voir un modèle de ce cheval incomplet au musée Fragonard de l'École nationale vétérinaire d'Alfort, daté de 1846, et un autre au Science museum de Londres.

Auzoux avait même prévu, dès 1844, de mettre en production trois modèles de $65 \mathrm{~cm}$ de haut, l'un complet, l'autre incomplet et le dernier simplement écorché ${ }^{30}$. Mais les faibles critiques émises en 1844 par les rapporteurs de l'Académie royale de Médecine furent reprises en $1847^{31}$ par le lieutenant-colonel Jacquemin, devenu promoteur de l'œuvre auprès de l'administration militaire. Le mannequin était en somme perfectible, et c'était là quelque chose qu'Auzoux ne pouvait supporter; il conçut donc un nouveau cheval de $1,30 \mathrm{~m}$ de hauteur, d'une qualité irréprochable, qui fut mis en vente au début des années $1850^{32}$ dans les deux versions, complet et incomplet, avec des tableaux synoptiques dédiés; chaque type bénéficiait dorénavant d'une numérotation des éléments qui lui était propre ${ }^{33}$. Ce cheval présentait le profil caractéristique des chevaux arabes, que les Français venaient de découvrir dans leur conquête de l'Algérie, en même 
temps que les pratiques de ces peuples cavaliers dont ils allaient faire leur nouvelle référence (fig. 7) $)^{34}$.

Figure 7

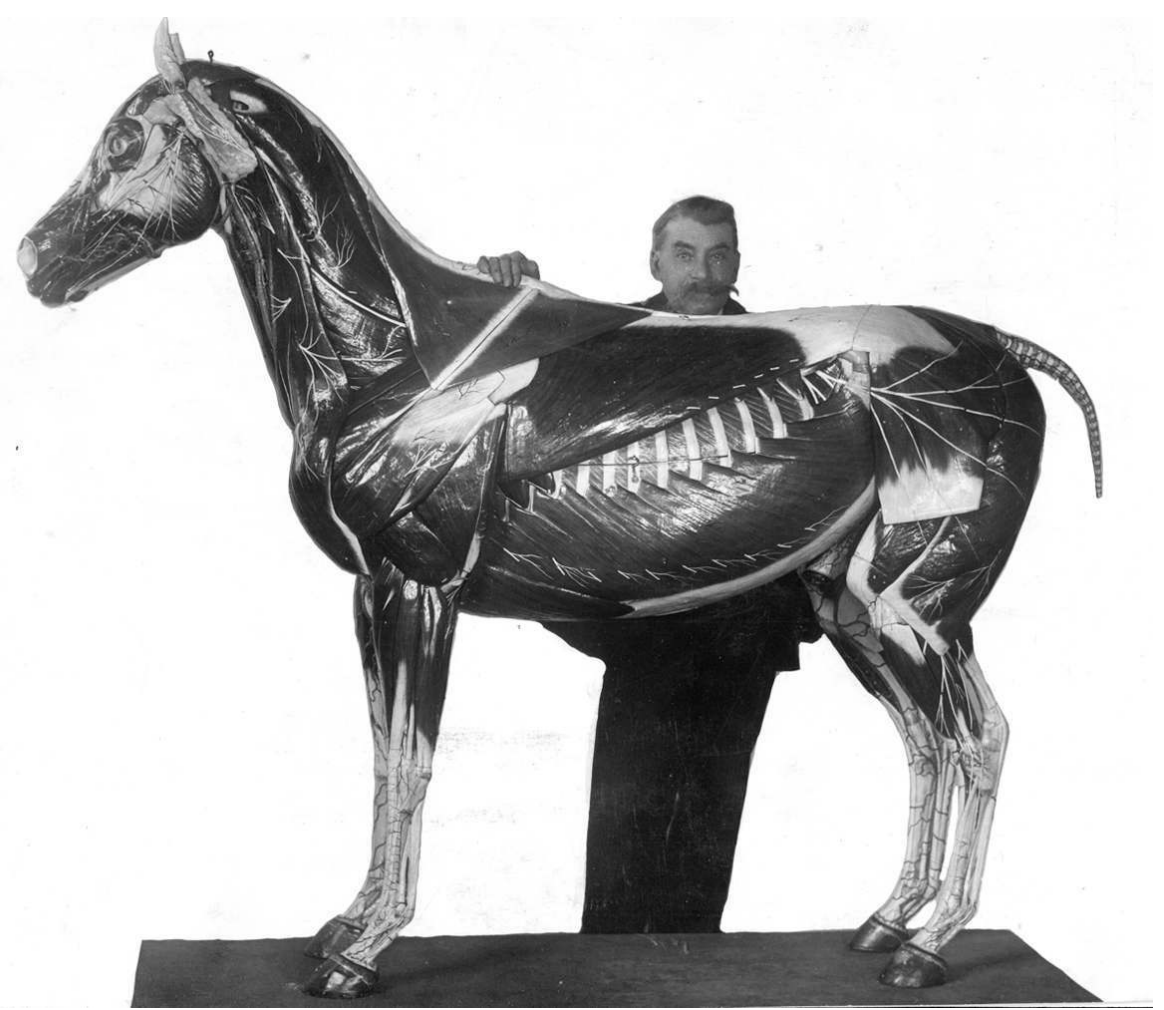

Un ouvrier posant à côté du cheval clastique, type arabe, au XXe siècle.

Repro. Degueurce, Christophe. (c) Musée de l'Écorché d'anatomie du Neubourg, juin 2012.

\section{Les autres modèles équins}

Mais il manquait des pièces complémentaires du cheval, indispensables pour éclairer le choix de l'animal lors de son achat par les autorités militaires, des objets que le célèbre général Oudinot ${ }^{35}$ recommanda à Auzoux de façonner dès 1845 . Tout d'abord, c'était une série de tares devant être détectées lors de la visite d'achat, formée au départ de cinquante modèles, dont le nombre ne cessa de croître avec les années (fig. 8). Auzoux fut notablement aidé par Jacquemin qui ne cessa de stimuler son ami, étudiant les pièces, les commentant, développant une énergie considérable que les correspondances du début des années 1850 attestent. Les pièces figurant les tares mimaient des pièces squelettiques et ligamentaires, mais Auzoux développa des pièces plus performantes. Partant du principe que ces lésions doivent être palpées à travers la peau, Auzoux conçut des modèles de membres, coupés au-dessus du jarret sur lesquels il était possible d'adapter des déformations qui étaient ensuite recouvertes par une peau naturelle, si bien que l'élève pouvait apprendre à palper les anomalies. Il fit notamment un ensemble qui devait connaître un grand succès, un triptyque formé d'un membre disséqué, d'un autre présentant les tares dures, osseuses, et d'un troisième affecté de tares molles, synoviales et tendineuses. 
Figure 8
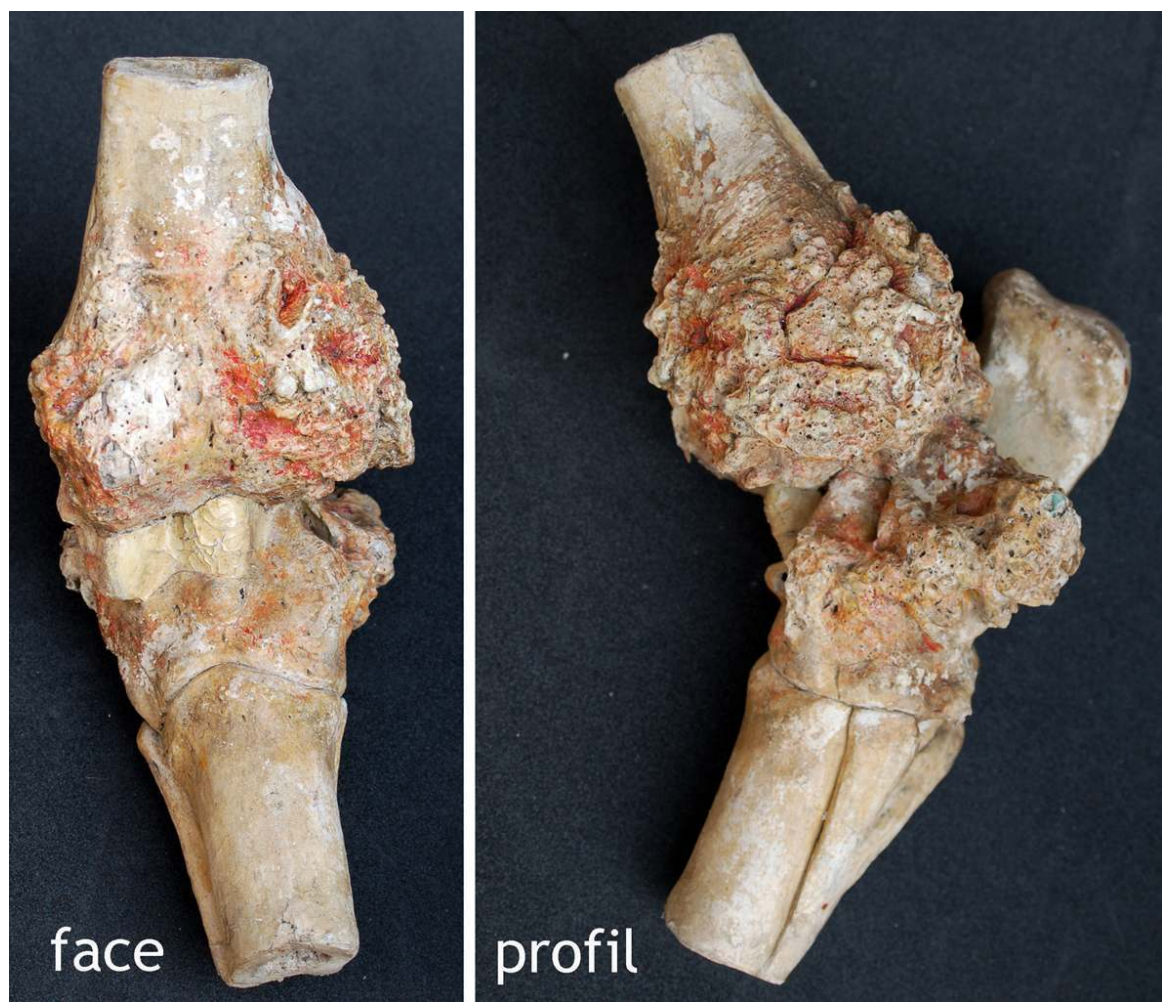

Un exemple de tare osseuse.

Phot. Ruiz, Guillaume. (c) Musée de l'École nationale vétérinaire de Maisons-Alfort, décembre 2009.

Il réalisa à la même époque une série de trente mâchoires de chevaux, copiées d'après des modèles naturels dont certains étaient issus des collections alforiennes ${ }^{36}$ et présentant plusieurs âges et les supercheries habituellement mises en œuvre par les maquignons pour tromper les acheteurs (fig. ${ }^{\circ}$ ). $)$. Rappelons que les responsables de ces achats étaient des officiers de cavalerie, assez peu versés dans ces nuances, et non des vétérinaires formés à ces notions dans les écoles vétérinaires. Auzoux s'était par ailleurs inspiré des traités en vogue, l'Hippélikiologie ou connaissance de l'âge du cheval de Narcisse Girard ${ }^{37}$ et surtout l'ouvrage de son ami Félix Lecoq ${ }^{38}$. La série, composée de trente mâchoires, présente notamment des arcades incisives artificiellement rajeunies, un procédé couramment utilisé par les maquignons pour vendre un cheval âgé ; l'astuce consistait à creuser une cavité dans les tables dentaires et la teinter avec de l'encre de Chine. 
Figure 9
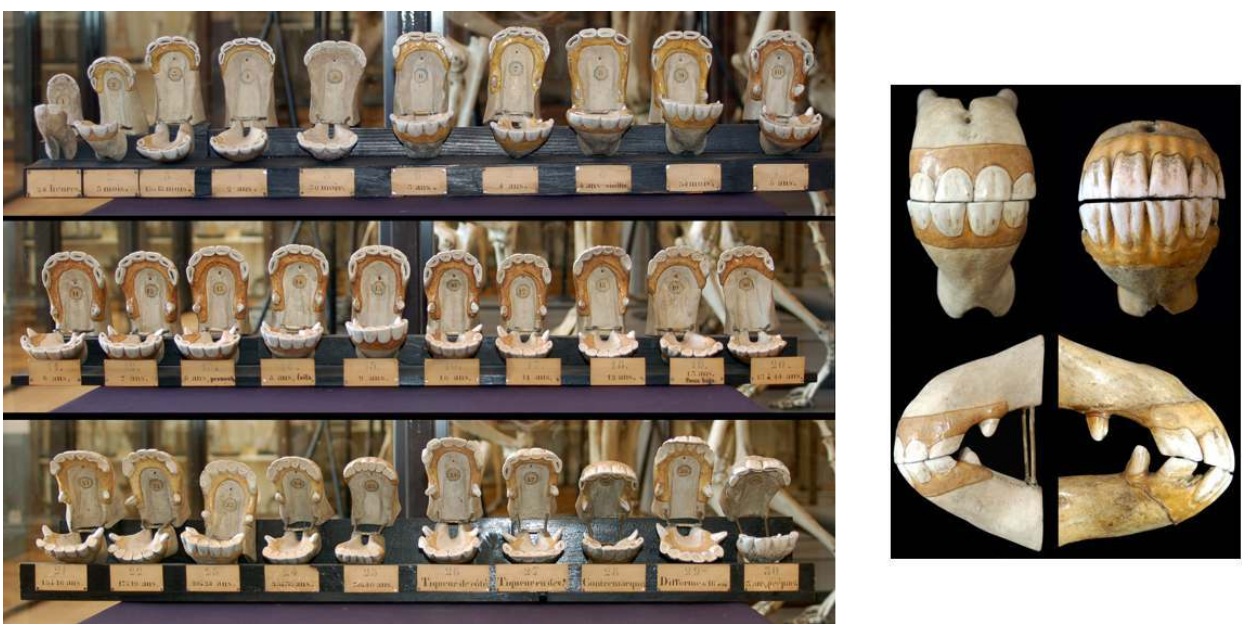

(a) La série des trente mâchoires créées par Auzoux. (b) Détails d'une des mâchoires.

Phot. Ruiz, Guillaume. (c) Musée de l'École nationale vétérinaire de Maisons-Alfort, décembre 2009.

21 Auzoux confectionna enfin deux pièces se rapportant au doigt du cheval, un organe conditionnant l'aptitude du cheval à son emploi. Il réalisa une anatomie complète du doigt du cheval, montrant les tendons, ligaments, os, gaines synoviales, vaisseaux et nerfs, entièrement clastique (fig. 10).

Figure 10

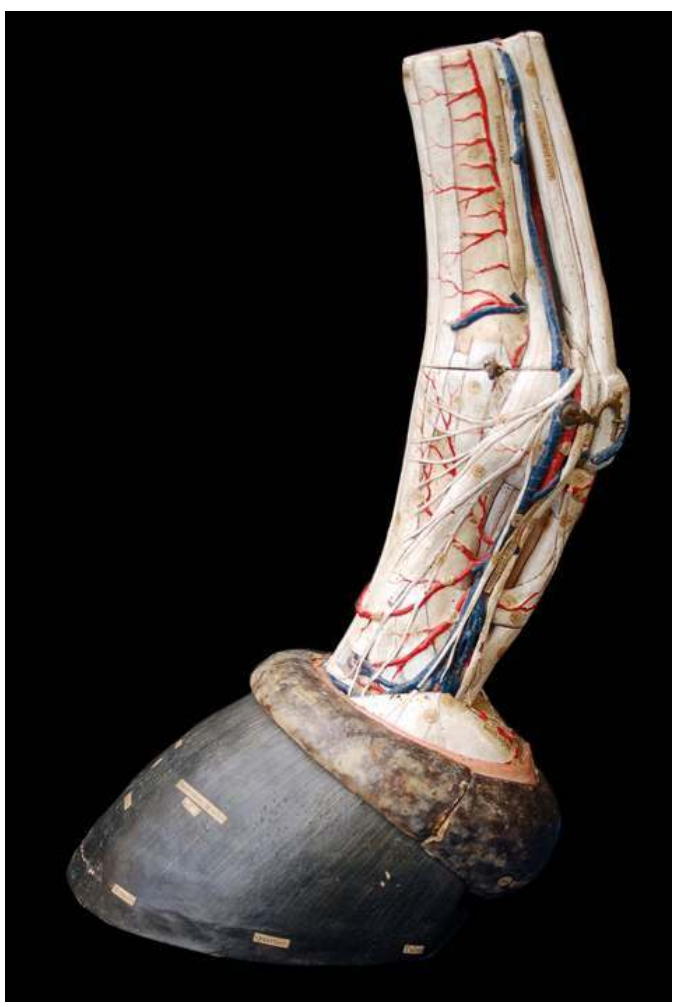

Le doigt du cheval.

Phot. Ruiz, Guillaume. (c) Musée de l'École nationale vétérinaire de Maisons-Alfort, décembre 2009. 
Il dédia ensuite une pièce au sabot, retranscrivant dans l'organisation des pièces la théorie sur l'élasticité du sabot édictée par le vétérinaire anglais Bracy-Clarck en $1817^{39}$. Ce dernier démontrait dans son ouvrage que le pied du cheval, à l'instar de celui des autres espèces, était déformable et que son élasticité permettait d'amortir les chocs. Cette notion était fondamentale car la ferrure pouvait permettre ou au contraire empêcher ces mouvements intimes. La conséquence d'une mauvaise gestion du pied était son rétrécissement, l'encastelure, la perte de son élasticité et ainsi la sollicitation anormale des autres formations anatomiques, osseuses et tendineuses en particulier. L'affaire se terminait généralement par une boiterie rendant l'animal impotent. Auzoux matérialisa dans la décomposition des pièces la théorie de l'auteur britannique (fig. 11).

Figure 11

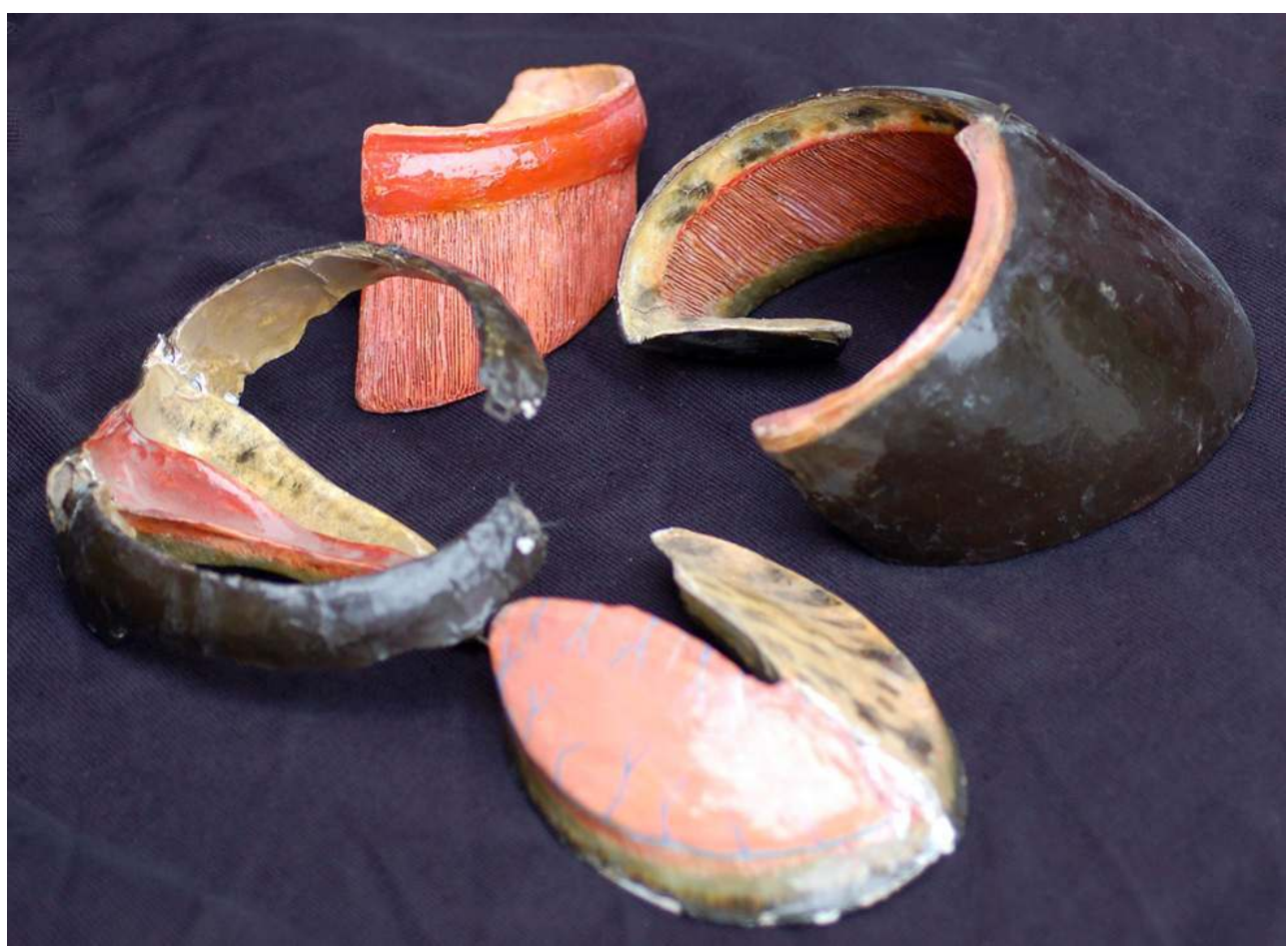

Le sabot à la façon de Bracy-Clarck.

Phot. Degueurce, Christophe. (C) Musée de l'École nationale vétérinaire de Maisons-Alfort, janvier 2013.

Enfin, et de façon périphérique, Auzoux créa quelques organes isolés de chevaux, destinés à illustrer les cours d'anatomie et de physiologie comparées qu'il dispensait à son domicile. C'est ainsi qu'il commercialisa un estomac fenêtré de cheval (fig. 12) et des appareils génitaux de jument. 
Figure 12

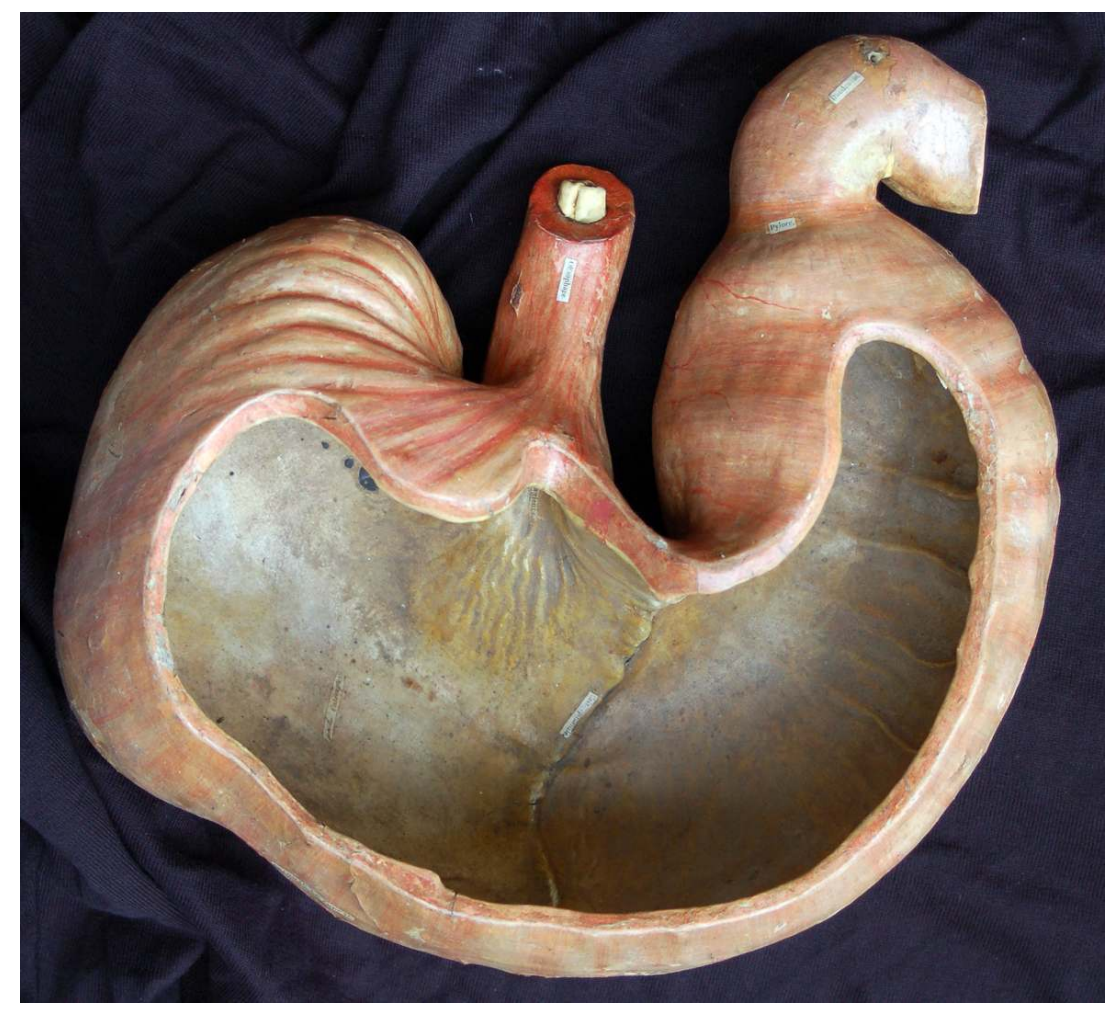

L'estomac du cheval.

Phot. Degueurce, Christophe. (C) Musée de l'École nationale vétérinaire de Maisons-Alfort, janvier 2013.

\section{Commercialisation et diffusion des modèles}

Le corollaire de la grande perfection des modèles de chevaux était leur prix extrêmement élevé, un défaut que le professeur d'anatomie d'Alfort, Rigot, lui signifiait avant même la commercialisation (fig. 13). Il l'alertait sur le risque qu'il prenait de se placer dans la même situation que le Traité de l'anatomie de l'homme de Jean-Baptiste Bourgery, magnifiquement illustré par Nicolas $\mathrm{Jacob}^{40}$, et qui peinait à se vendre. Auzoux risquait d'affronter les mêmes difficultés que celles qui avaient limité la diffusion de son homme complet d'1,80 m. Il avait en effet fortement peiné à le faire acheter par les facultés médicales et n'était parvenu à ses fins qu'au prix de très nombreuses relances et interventions des pouvoirs publics. Il n'y avait guère que les ministères de la Guerre d'une part, et du Commerce et de l'Agriculture d'autre part qui pouvaient acquérir des pièces aussi onéreuses. 
Figure 13

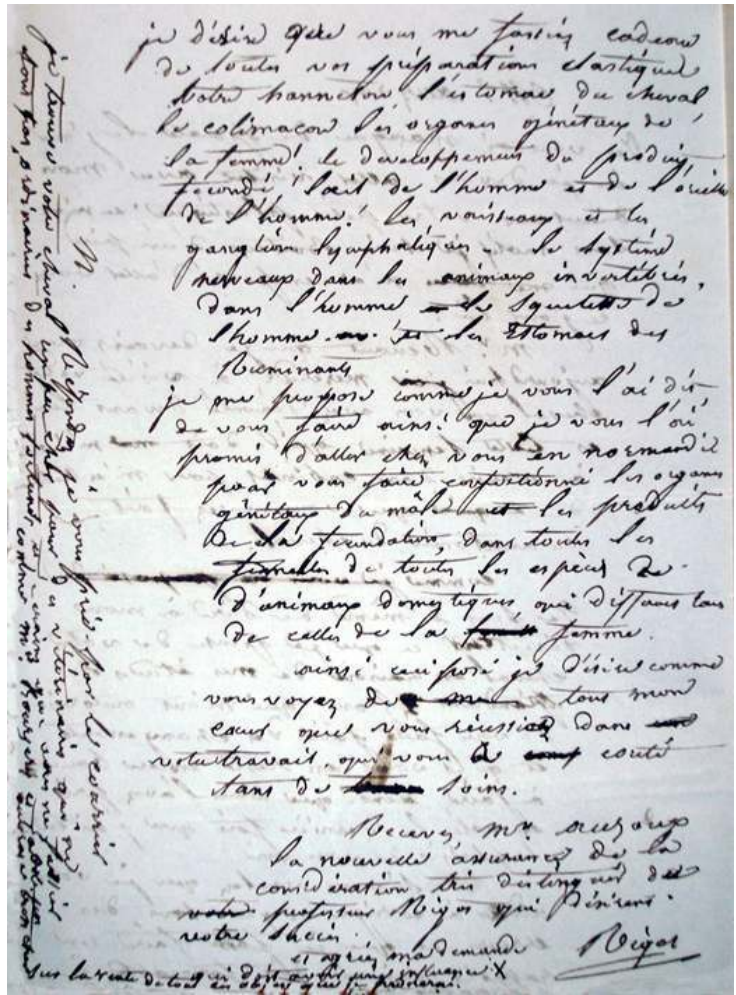

Lettre de Rigot à Auzoux, probablement de 1845.

Repro. Degueurce, Christophe. (c) Archives nationales, juin 2012.

Du côté militaire, la chose s'engageait bien car l'enseignement de l'hippologie était rendu obligatoire par l'administration à l'École royale de cavalerie, dans les dépôts de remonte et dans tous les corps de troupes à cheval. Le programme avait été défini par une commission compétente en 1825 et matérialisé par un ouvrage à caractère officiel, le Cours d'équitation militaire à l'usage des corps de troupes à cheval, publié par $1830^{41}$. Le cheval clastique présentait le grand intérêt d'apporter un support à cet enseignement sinon aride. Le 21 juillet 1845, le ministre décidait, d'après l'avis du comité de Cavalerie, qu'un cheval artificiel serait placé à l'École royale de cavalerie de Saumur et dans les dépôts de remonte de Caen, Villers, Guingamp, Saint-Maixent Guéret et Auch ${ }^{42}$ (fig. 14), le coût devant être prélevé sur les fonds alloués au harnachement et à la ferrure. 


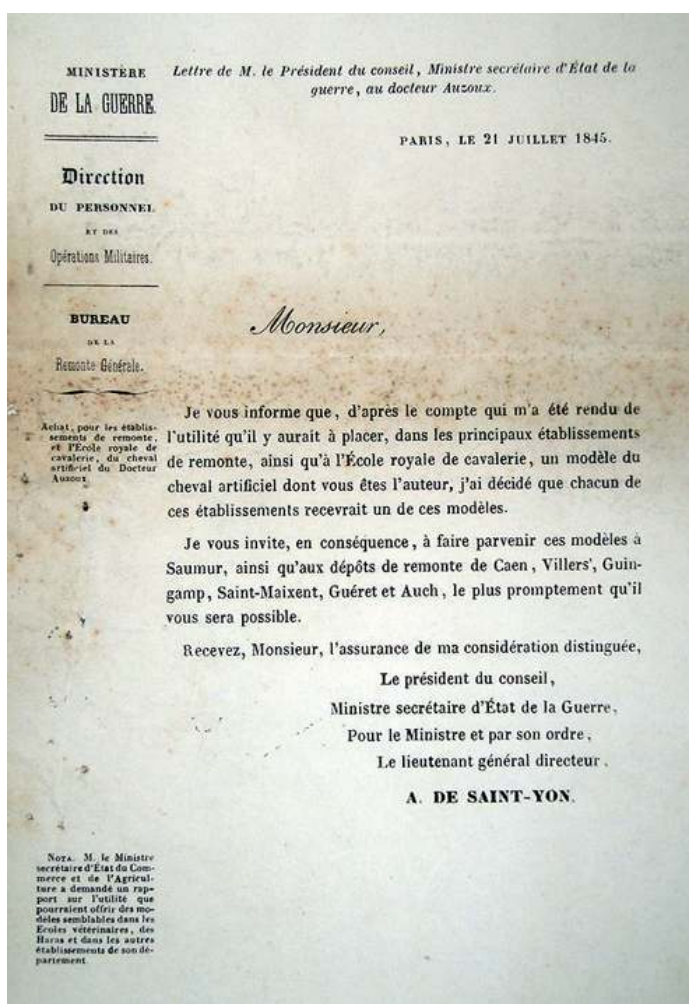

Lettre du service des remontes à Auzoux, datée du 21 juillet 1845, l'invitant à pourvoir l'École de Saumur et quelques dépôts de remonte de son précieux cheval clastique.

Repro. Degueurce, Christophe. (c) Archives nationales, juin 2012.

Quant au secrétariat d'État au Commerce et à l'Agriculture, il engageait dans le même temps une réflexion sur l'opportunité de doter les écoles vétérinaires, les haras et les autres écoles dépendant de son administration du modèle, ce qui allait se traduire dans les mois qui suivirent par l'attribution d'un spécimen à l'École des haras et de l'agriculture ${ }^{43}$. Souhaitant amplifier le mouvement, Auzoux se chargeait lui-même de promouvoir sa pièce auprès des commandants des régiments de cavalerie, leur envoyant des lettres circulaires attirant leur attention sur l'intérêt de son cheval et des mâchoires et tares qu'il venait de créer ${ }^{44}$. Mais il y avait encore un autre débouché : les régiments d'artillerie, dont le train utilisait un très grand nombre de chevaux. Le 3 mai 1847, le comité de l'Artillerie rendait un avis positif qui engageait le ministre de la Guerre à faire placer dans les écoles d'artillerie le cheval clastique, ainsi que les mâchoires et les tares osseuses $^{45}$.

Comme chaque fois, Auzoux allait amplifier la promotion de ses créations. En 1847, il publiait un petit livret de douze pages intitulé De l'utilité de l'anatomie clastique sous le rapport du choix, de l'emploi, de la conservation du cheval ${ }^{46}$ visant à montrer son intérêt pour la formation des élèves officiers et l'amélioration de la condition du cheptel équin de la France du XIXe siècle (fig. 15). Son ami Jacquemin complétait l'opuscule par un Compte rendu de l'Anatomie clastique du Dr Auzoux et de l'influence qu'elle doit avoir sur l'Instruction de la Cavalerie ${ }^{47}$. L'ensemble visait à convaincre les décideurs et à pallier l'incapacité dans laquelle il était de leur présenter de visu une pièce peu déplaçable car aussi imposante que fragile. L'argumentaire d'Auzoux montrait comment ses collections permettaient, en 
aidant à former mieux l'officier de cavalerie, d'améliorer le choix du cheval en commission d'achat, d'en optimiser ensuite l'emploi et d'en assurer une conservation optimale. Auzoux jouait alors sur la corde sensible car l'administration militaire avait encore bien à l'esprit la perte de dizaines de milliers de chevaux en 1808 en Espagne et en 1812 en Russie, du fait de la mauvaise gestion de leur alimentation ${ }^{48}$. Il allait s'avancer plus avant et décrire la situation catastrophique de l'élevage équin français, qui plaçait l'administration des remontes ${ }^{49}$ dans une situation de grande précarité, sujet qui était alors mis en exergue par la polémique déclenchée par le général Oudinot en 1842. Le déficit chronique de la production de chevaux de qualité rendait la France dépendante des pays voisins, ce qui constituait un problème financier et un risque stratégique. Il fallait améliorer la production équine et surtout rationaliser l'élevage et la mise au travail du jeune cheval, des domaines où les pratiques françaises étaient arriérées et préjudiciables à toute la filière; la France produisait bien les 300000 poulains annuellement nécessaires mais l'incohérence de leur élevage les rendait impropres à tout usage dès les premières années de leur vie.

\section{Figure 15}

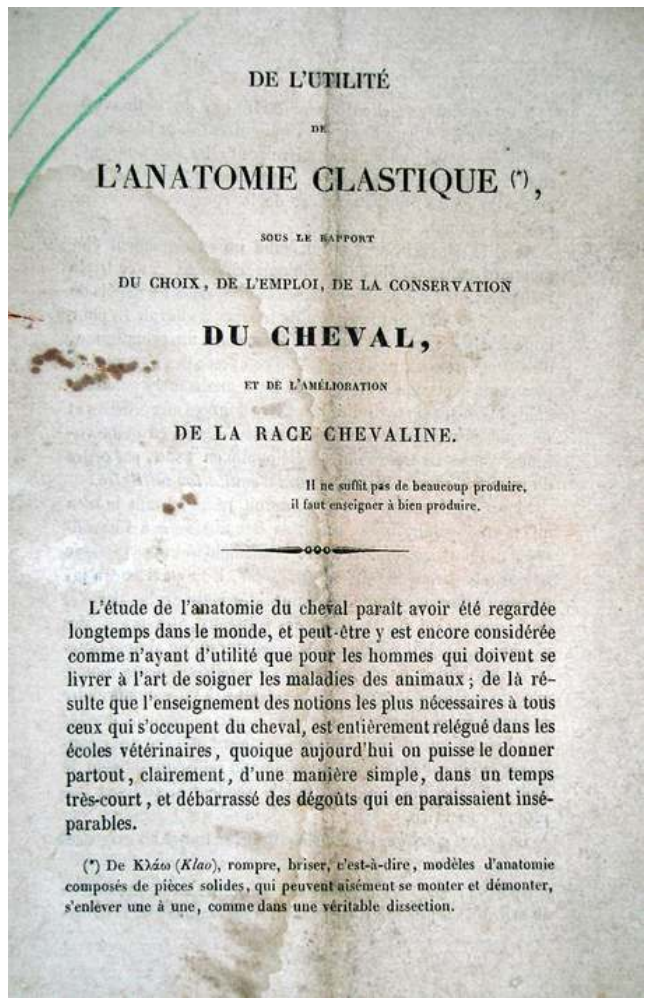

Livret publié en 1847 par Auzoux et largement diffusé par ce dernier pour assurer la promotion de son cheval clastique.

Repro. Degueurce, Christophe. (c) Archives nationales, juin 2012.

Auzoux, en républicain convaincu, croyait à la force de l'éducation ${ }^{50}$. Il proposait donc dans sa brochure de promouvoir un processus analogue à celui de la mérinisation des troupeaux ovins. L'élevage du mouton dans la France de la fin de l'ancien régime était dans un état de grande dégradation et des importations de mérinos avaient été tentées plusieurs fois, sans succès, depuis 1666 ; il avait fallu la création d'une école de bergers par Jean-Marie Daubenton en 1786 pour que de nouvelles méthodes soient enseignées à de jeunes gens et largement diffusées par eux dans une paysannerie rebelle à 
l'innovation. Si l'on voulait produire des chevaux de belle qualité, il fallait former des éleveurs de chevaux et leur enseigner la science du cheval, les faire pénétrer au cœur de la machine pour qu'ils sachent choisir l'animal, l'élever correctement, dans le respect de ses aptitudes et de ses limites, détecter une pathologie naissante et la juguler. Cette démarche était renforcée par la publication, la même année, de l'ouvrage de Richard qui développait le parallèle entre le premier échec de la mérinisation du mouton et celui que connaissaient les haras pour le cheval ${ }^{51}$ et arguait de l'intérêt fondamental de développer une gestion raisonnée des races, à l'instar de ce que les bovins connaissaient alors avec la durhamisation $^{52}$. Il fallait éduquer la population pour que la production du cheval soit rationalisée et devienne efficace.

Cette idée n'allait cesser d'obséder Auzoux. Il continuait son œuvre inlassable de persuasion en publiant en 1854 Insuffisance, en France, du Cheval de Guerre et de luxe. Possibilité de l'obtenir en créant dans les Régiments de cavalerie des Écoles d'éleveurs au moyen du Cheval clastique du Docteur Auzoux ${ }^{53}$, un argumentaire plus convaincant encore sur le dispositif à mettre en place autour de son cheval clastique pour réformer l'élevage français. Le discours reprenait celui de sa brochure de 1847 mais se faisait plus précis : « les 7 ou 8000 libérés chaque année iraient au centre de la production chevaline, c'est-àdire dans les fermes, porter l'art d'améliorer les races ». Pour convaincre, il dispensait luimême des leçons aux éleveurs et propriétaires de chevaux, comme ce fut le cas lors de l'Exposition générale et nationale d'Agriculture de juin 1860. Et il reprenait ce discours dans ses Leçons élémentaires d'anatomie et de physiologie humaine et comparée publiées en 1858, réédition de son Cours de physiologie anatomique de 1839, qu'il illustra de gravures représentant son cheval à différents niveaux de démontage. "Faisons des écoles d'éleveurs de chevaux » s'exclamait-il alors ${ }^{54}$.

Un lieu réunissait de façon obligée ces futurs disciples: les régiments où les jeunes faisaient leur service militaire. Ainsi Auzoux avait-il créé son modèle "incomplet » dans le but de le placer dans les écoles et les régiments de cavalerie, dans les régiments d'artillerie et dans les dépôts de remonte. Il théorisait ainsi un enseignement global, s'appuyant sur l'administration militaire et visant à améliorer un élément stratégique de l'économie française, tout ceci pour un coût modéré puisqu'il ne fallait que développer un peu les notions d'anatomie, de physiologie et d'hygiène déjà professées dans le cours d'équitation militaire et, pour rendre les leçons plus démonstratives, s'appuyer sur... son cheval clastique ${ }^{55}$. Le discours était convaincant et Auzoux allait le réitérer dans plusieurs brochures consacrées aux collections de tares en $1848^{56}$ et $1853^{57}$, ou aux mâchoires en $1850^{58}$.

31 Le résultat fut à la hauteur de ses attentes. L'École de cavalerie de Saumur, le phare de la cavalerie française, recevait son modèle dès juillet $1845^{59}$. Et la même École commandait la collection de tares osseuses, l'anatomie du pied et le sabot à la façon de Bracy Clarck en octobre $1851^{60}$. Surtout, en 1853, l'armée commandait «soixante-huit exemplaires complets du cheval artificiel $\|^{61}$, livrables à raison de douze exemplaires par an et un nombre beaucoup plus important de mâchoires et de tares. D'autres spécimens vinrent peupler les régiments d'artillerie, à l'instar de celui de Douai en $1848^{62}$.

32 Le résultat fut en revanche un peu moins probant du côté de l'agriculture. Il tenta vainement de transposer l'idée qu'il avait développée dans l'administration militaire au niveau des départements, invitant en 1860 les conseils généraux à créer une école d'agriculture dans chaque département afin que des enseignements soient dispensés aux éleveurs, charretiers, à tous les acteurs de l'élevage du cheval en somme, en les attirant 
par la délivrance d'un diplôme ${ }^{63}$. Le ministre de l'Agriculture soutint son initiative; en août 1860, il sollicitait les préfets par une lettre circulaire pour qu'ils poussent les conseils généraux à acheter le cheval clastique ${ }^{64}$. Il réussit tout au plus à faire acquérir quelques modèles à des administrations relevant de l'agriculture: l'École des haras reçut son modèle dès $1846^{65}$, l'École vétérinaire d'Alfort, qui avait été partenaire du projet, recevait également très rapidement son modèle puis l'échangeait contre le nouveau modèle en juin $1856^{66}$; elle achetait la série des mâchoires en janvier $1851^{67}$. L'École vétérinaire de Lyon recevait les mâchoires au début de 1851 puis son modèle complet de cheval en décembre $1851^{68}$, où il se trouve toujours.

Mais tous ces modèles connurent un succès mondial et furent diffusés sur les cinq continents, où on les trouve encore. Le visiteur français est surpris en visitant tel musée de New Delhi d'y découvrir un cheval qui a fait le long voyage au XIX ${ }^{\text {e }}$ siècle, ou encore de découvrir une importante collection au Powerhouse museum de Sydney, preuve que le succès fut planétaire ${ }^{69}$.

Les modèles équins d'Auzoux eurent ainsi une grande importance pour l'entreprise économique du Dr Auzoux et répondirent à un besoin évident de la France; il suffit de constater qu'Auzoux fut promu en 1862 au grade d'officier de la Légion d'honneur au titre du génie et du service des remontes comme «inventeur des appareils clastiques d'anatomie en usage dans l'armée et les écoles militaires $\aleph^{70}$ pour saisir l'apport qui a été le sien.

\section{Des pièces fragiles, dégradées, dispersées, menacées de disparition}

Toutes ces pièces étaient parfaitement utilitaires et beaucoup sollicitées. Elles étaient continuellement démontées et remontées au gré des enseignements, si bien qu'elles s'abîmaient, d'autant qu'elles étaient faites de liège et de papier, des matériaux fragiles. Dès 1848, Jacquemin demandait par exemple à Auzoux qu'il lui fasse

parvenir une feuille des numéros et des mains indicatrices qui vous servent pour numéroter les différentes pièces de votre cheval. Par suite d'un usage fréquent, plusieurs de ces marques se sont détachées et perdues, et il importe de les remplacer promptement, pour prévenir des erreurs et conséquemment des dégradations dans le remontage ${ }^{71}$.

À ces dégradations s'ajoutèrent les spoliations, par le vol, par l'armée prussienne lors de son retrait en 1871, à moins qu'elles aient été opportunément chapardées par quelques Français à l'occasion de cette confusion. Le ministère de la Guerre sollicitait en 1874 un devis de Auzoux pour la livraison d'" environ" cent dix caisses contenant chacune la série des trois jambes avec les tares et l'anatomie du doigt ${ }^{72}$.

In fine, il est très difficile de se faire une idée précise de l'ampleur de la production de cette entreprise. Nous avons cherché à établir en 2012 la liste exhaustive de ces modèles et force est de constater qu'alors qu'on considérait que 100 à 150 modèles avaient été produits, le nombre réel est probablement supérieur à 300 objets si on considère que certains ensembles, figurés par un numéro d'ordre dans un catalogue, sont constitués de 30 à 50 objets. Ces pièces sont aujourd'hui dispersées dans nombre de collections et ont des statuts très variables. Elles sont protégées lorsqu'elles sont placées chez des amateurs ou dans des musées; elles sont en revanche souvent délaissées dans les collections utilitaires, militaires ou éducatives. La plupart des régiments, des collèges et lycées déjà 
existants au XIX ${ }^{\mathrm{e}}$ siècle avaient de vastes collections de modèles d'Auzoux. Pour la plupart, elles n'existent plus ou ont été profondément dégradées par les démontages partiels, l'empoussièrement ou les variations climatiques subies dans des locaux inadaptés.

Ces moulages sont particulièrement fragiles ${ }^{73}$. Fait d'une pâte moulée qui forme les parties du modèle, chaque élément était ensuite recouvert d'un papier d'habillage, peint finement ${ }^{74}$ et vernis au moyen d'une colle de poisson (fig. 16). Ce dernier revêtement absorbe aisément la poussière qui noircit ainsi les surfaces, et ceci d'autant plus que la température est élevée et la gélatine ramollie. Les variations d'hygrométrie mettent en œuvre des contraintes qui entraînent le décollement des couches superficielles : la couche picturale se désolidarise du papier sous-jacent et forme des écailles qui tombent facilement et créent des manques. La lumière affecte les protéines présentes dans les couches de surface. Surtout, la pièce, lorsqu'elle est laissée démontée, se déforme et ne peut plus être remise en place. Dès lors, les tiges métalliques et les crochets ne retrouvent plus leurs logements et bien souvent les utilisateurs, en forçant, causent des dommages importants.

Figure 16

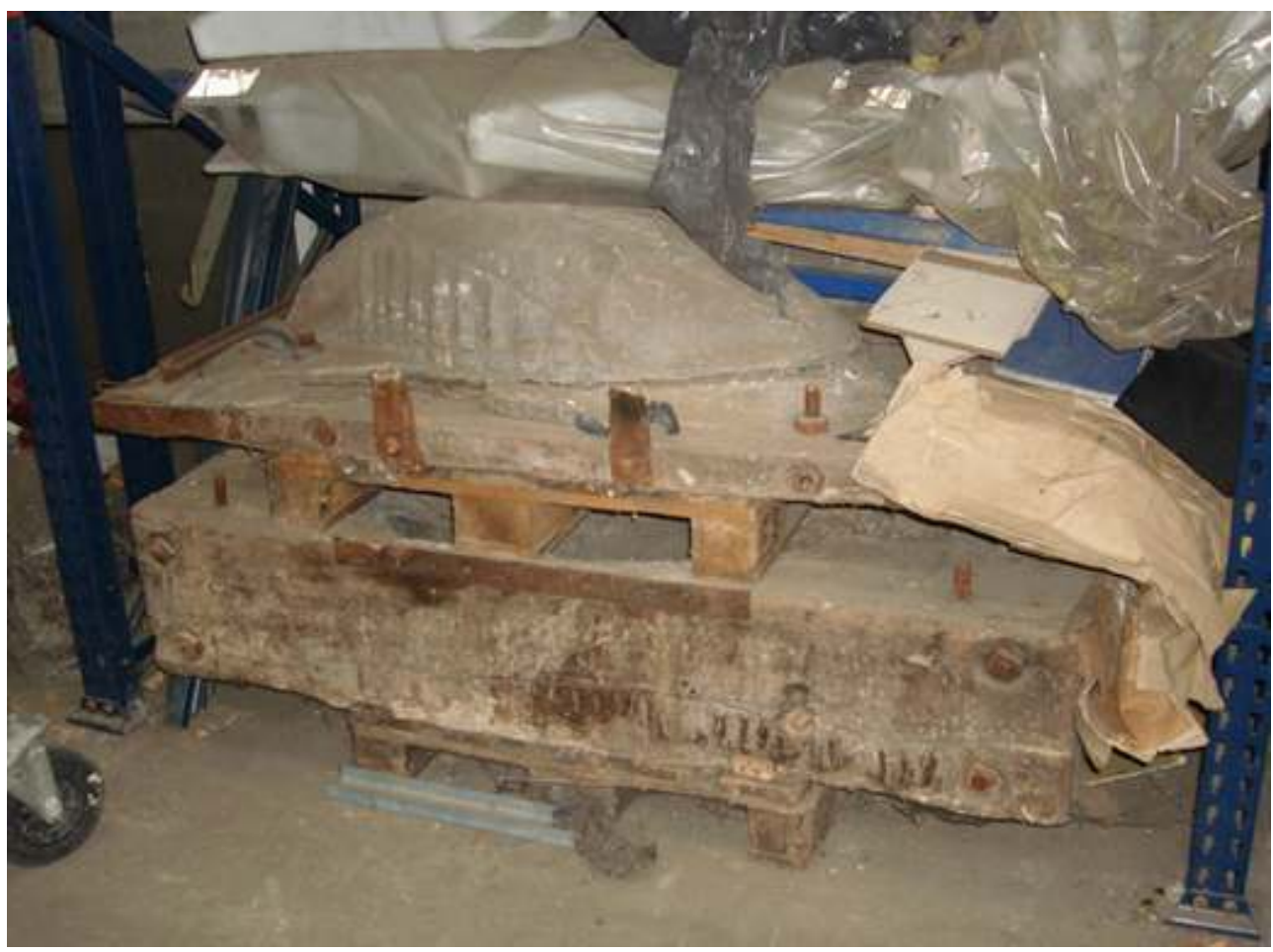

Les moules du cheval, conservés au Neubourg.

Phot. Degueurce, Christophe. (c) Musée de l'Écorché d'anatomie du Neubourg, juin 2012

Au regard de l'importance historique, culturelle, économique de ces objets du patrimoine français, il semble indispensable de mettre en œuvre une politique raisonnée de sauvegarde de ce patrimoine national si connu à l'étranger. Cela passe par le recensement des objets présents sur le sol français, l'information des gestionnaires des collections identifiées et la mise en place de mesures de conservation préventive de ces pièces. Le développement d'un catalogue raisonné et, en phase intermédiaire peut-être, la création d'un musée virtuel, constituent une seconde phase (fig. 17). Enfin, s'il est permis de 
rêver, le rassemblement dans un même lieu de la collection permettrait d'envisager la mise en œuvre des coûteux processus de restauration et la mise en place d'une médiation consacrée à l'ensemble de la production d'Auzoux. À cet égard, le musée de l'Écorché d'anatomie du Neubourg (Eure), sous réserve d'une professionnalisation de la conservation, pourrait légitimement être le centre de ce dispositif. Situé à proximité des établissements Auzoux, il rassemble déjà les souvenirs de cette industrie locale et présente au visiteur un grand nombre de modèles confectionnés dans l'usine voisine, ceci n'excluant pas que d'autres musées abordent tel ou tel ensemble thématique qui ressort de leur spécialité.

Figure 17

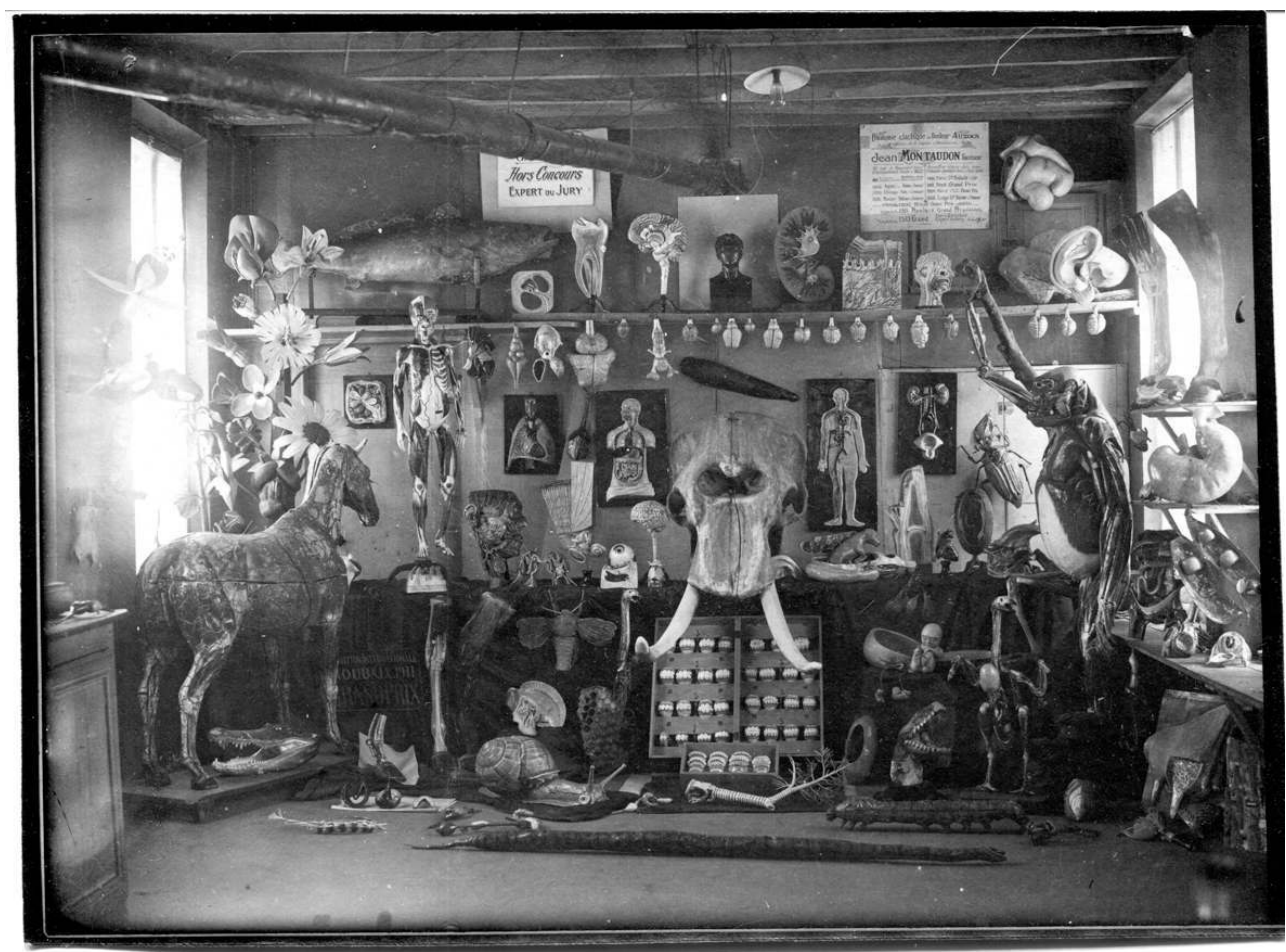

La production des établissements Auzoux vers 1910. On distingue le cheval à gauche; juste à droite et au sol, la jambe de cheval écorchée et la même avec la peau, le doigt clastique et le sabot à la Bracy-Clarck ; sous la tête d'éléphant au centre, les mâchoires dans leur boîte de conditionnement ; sur les étagères à droite, l'estomac et, au-dessus, à nouveau les jambes.

Repro. Degueurce, Christophe. (c) Musée de l'Écorché d'anatomie du Neubourg, juin 2012.

40 L'auteur engage toutes les personnes intéressées par ces collections à se rendre au musée de l'Écorché d'anatomie situé au Neubourg (Eure) ${ }^{75}$.

41 Enfin, ceux qui voudraient en savoir plus sur Auzoux et la très grande étendue de sa production peuvent consulter :

42 DEGUEURCE, Christophe. Corps de papier: l'anatomie en papier mâché du docteur Auzoux. Paris : La Martinière, 2012. 


\section{NOTES}

1. - Ce terme désignait au XVIII ${ }^{\mathrm{e}}$ siècle les collectionneurs.

2. - DEGUEURCE, Christophe. «Éloge des matières ». Dans DEGUEURCE, Christophe et DELALEX, Hélène. Beautés intérieures, l'animal à corps ouvert. Paris : Éditions de la Réunion des Musées Nationaux, 2012, p. 80.

3. - DEGUEURCE, Christophe. Corps de papier : l'anatomie en papier mâché du docteur Auzoux. Paris : La Martinière, 2012.

4. - AMELINE, Jean-François. Observations sur les pièces d'anatomie de M. le docteur Auzoux. Caen : Bonneserre, 1825, p. 5.

5. - « Rapport fait par M. Béclard, Duméril, Hippolyte Cloquet, Breschet, Desgenettes sur une pièce d'anatomie artificielle de $\mathrm{M}$. Auzoux, représentant le pied, la jambe, la cuisse et une partie du bassin. Séance du 5 novembre 1823, rapport publié en février 1824 ». Dans AUZOUX, Louis. Notice sur les préparations artificielles de M. Auzoux. Paris : chez l'auteur, 1825, p. 13.

6. - « Rapport fait par une commission nommée pour examiner une pièce d'anatomie imitative de M. Auzoux, destinée à représenter la tête, le cou et la partie supérieure du tronc, par M. Worbe, Bégin et Desruelles ». Dans AUZOUX, Louis. Notice sur les préparations artificielles de M. Auzoux. Paris : chez l'auteur, 1825, p. 17-19.

7. - «Clastique » : Dictionnaire de la conversation et de la lecture. Tome 14. Paris : Belin, 1834, p. 453.

8. - Écuyer dans ladite école, École de cavalerie de Saumur.

9. - Un écorché de cheval goudronné se trouve encore au Centre sportif d'Équitation militaire de Fontainebleau. Il est, à notre connaissance, le dernier représentant de cette technique anatomique.

10. - RENAULT, Eugène. «Rapport fait par M. Renault, professeur et directeur de l'École royale vétérinaire d'Alfort, à l'Académie royale de médecine dans sa séance du 22 juillet 1845 ». Recueil de Médecine Vétérinaire, 1845, p. 843-851.

11. - Fonds Montaudon, Archives nationales 242API.

12. - LAFOSSE, Philippe Étienne. Cours d'Hippiatrique. Paris : Edme, 1772.

13. - Auzoux a dû vouloir dire "conformation », le traité en question étant un traité d'extérieur du cheval tout récemment paru : LECOQ, Félix. Traité de l'Extérieur du cheval. Paris, Vve BouchardHuzard, 1843.

14. - Félix Rigot, 1803-1847. Il avait été nommé professeur d'anatomie de l'École royale vétérinaire d'Alfort en 1838 et avait publié les articulations en 1840, les os en 1841, les muscles en 1842. Il publiera la première partie de l'angéiologie en 1845, trop tard donc pour qu'Auzoux pût en bénéficier.

15. - AUZOUX, Louis. Leçons élémentaires d'Anatomie et de Physiologie humaines et comparées. Paris : Labé et Dumaine, 1858, p. XIV.

16. - Eugène Renault, 1805-1863, avait fait ses observations à Auzoux le 25 avril 1845 [Note manuscrite de Louis Auzoux. AN 242API/2]. Il était alors directeur de l'École royale vétérinaire d'Alfort; il devint inspecteur général des écoles vétérinaires françaises en 1860 et mourut en 1863.

17. - Armand Goubaux, 1819-1890. Il fut nommé professeur d'anatomie à l'École vétérinaire d'Alfort en 1846, directeur en 1879 et prit sa retraite en 1888. Son nom est resté attaché au Traité d'extérieur du cheval qu'il publia avec son successeur, Gustave Barrier, en 1884.

18. - Rigot avait pu faire ses observations à Auzoux le 20 avril 1845 [Note manuscrite de Louis Auzoux (AN 242API/2)]. Les billets de correspondance conservés aux Archives nationales 
montrent que le pauvre homme, atteint par une maladie neurologique, était diminué. Il se retirait la même année et décédait en 1847.

19. - Antoine Richard, 1802-1891, fut élève militaire à l'École d'Alfort avant de devenir vétérinaire au $1^{\mathrm{er}}$ régiment d'artillerie stationné à Strasbourg. C'est là qu'il suivit les cours de la faculté de médecine et obtint le titre de docteur en médecine. Il fit la campagne d'Algérie avant de devenir professeur d'économie rurale à l'École de Grignon en 1838, puis professeur à l'École des haras en 1840, établissement dont il prit la direction en 1845. Destitué de son poste en 1847, il commença une carrière politique dans le camp des républicains. Il fut envoyé à Saint-Flour comme sous-commissaire du gouvernement après les événements de 1848, et élu la même année représentant du Cantal à l'Assemblée constituante. Élu en 1849 à la Législative, il fut rendu à la vie privée par le coup d'État de 1851. Un temps écarté, ses compétences lui valurent d'être nommé inspecteur général des Haras.

20. - RICHARD, Antoine. De la Conformation du Cheval selon les lois de la Physiologie et de la Mécanique. Paris : Guiraudet et Jouaust, 1847.

21. - RICHARD, Antoine. Étude du Cheval de service et de guerre suivant les principes élémentaires des Sciences naturelles. Paris : Guiraudet et Jouaust, 1859.

22. - Maxime Jacquemin, 1795-1863, était un officier de cavalerie en vue. Il avait notamment fait carrière à Saumur où il avait été sous-écuyer en 1828, capitaine instructeur en 1830, lieutenantcolonel en 1845, avant de commander en second l'École de cavalerie de Saumur à partir de 1848, puis finalement d'être promu au grade de général de brigade et d'être placé en poste à ClermontFerrand en 1852.

23. - Ce n'est pas le sujet ici d'expliquer pourquoi cohabitaient deux systèmes de formation aux soins des chevaux, celui des vétérinaires militaires formés dans les écoles du même nom, et celui des officiers de cavalerie, qui avaient autorité hiérarchique sur lesdits vétérinaires, et se mêlaient aussi de ces questions-là.

24. - Cette abondante correspondance est visible aux Archives nationales (242API). On y lit l'obstination de Jacquemin à faire réaliser ou améliorer par Auzoux les fameuses tares (années 1850 et 1851).

25. - La méthode de confection était identique à celle des autres pièces. Des moules permettaient de former les différentes pièces, qui étaient ensuite ajustées, assemblées, peintes et légendées.

26. - Pour les personnes souhaitant en savoir plus sur la technologie de ce modèle, l'auteur les engage à consulter : DUMONT, Barbara. Conservation-restauration d'un Écorché de cheval en carton moulé et peint, dit Cheval clastique, de Louis Auzoux. Mémoire de fin d'études du diplôme de restaurateur du patrimoine dans la spécialité sculpture. Paris : Institut national du Patrimoine, 2008.

27. - La démarche n'était pas nouvelle : Auzoux accompagnait chaque modèle de ce fascicule.

28. - AUZOUX, Louis. Tableau synoptique du cheval [complet]. Paris : Labé, 1845.

29. - AUZOUX, Louis. Tableau synoptique du cheval [incomplet]. Paris: Labé, 1855.

30. - LEQUIME, J.E. «Exposition des produits de l'industrie nationale en France ». Archives de la médecine belge, 1844, p. 412-415. Ces trois modèles devaient coûter respectivement, 2 000, 1000 et 500 francs, c'est-à-dire entrer dans la gamme des modèles humains de $1 \mathrm{~m} 16$ et $82 \mathrm{~cm}$ de haut.

31. - JACQUEMIN, Maxime. Compte rendu de l'anatomie clastique du Dr Auzoux et de l'influence qu'elle doit avoir sur l'Instruction de la Cavalerie. Paris : Firmin-Didot, 1847, p. 5.

32. - Louis Auzoux ne semble pas avoir fait de publicité sur le remplacement de son modèle de cheval; peut-être ne voulait-il pas attirer l'attention des acheteurs sur les imperfections du premier modèle? Il est donc assez difficile de dater de façon parfaite sa création. Il n'apparaît qu'une fois dans les archives de Louis Auzoux, dans une liste manuscrite des modèles créés où figure le «cheval type arabe » (AN 242API/2). Ce mannequin apparaît dans le catalogue inséré dans l'ouvrage sur les tares dures et les tares molles qu'il publia en 1853, et Auzoux livre le cheval du premier moule à l'École vétérinaire de Lyon en décembre 1851. On peut donc considérer qu'il a 
été créé en 1852 ou 1853. L'École d'Alfort a échangé son premier modèle contre le second en 1856. Il n'a pas, à ce jour, été retrouvé d'exemplaire de ce second moule sur le territoire français; il est visible dans les collections de l'université de Halle en Allemagne.

33. - AUZOUX, Louis. Tableau synoptique du cheval. Paris : Labé, 1855.

34. - Cet engouement est particulièrement perceptible dans les ouvrages suivants : DAUMAS, Melchior. Les Chevaux du Sahara. Paris: Chamerot, 1851. AUZOUX, Louis. Leçons élémentaires d'anatomie et de physiologie humaine et comparée. Paris : Labé, 1858. RICHARD, Antoine. Étude du Cheval de service et de guerre suivant les principes élémentaires des Sciences naturelles. Paris : Hachette, 1859.

35. - Nicolas Oudinot de Reggio, 1791-1863, fils aîné du maréchal de Reggio, fut page de l'Empereur en 1805, lieutenant en 1809 et colonel en 1814. Devenu maréchal de camp en 1824, il commanda l'École royale de cavalerie de Saumur de 1824 à 1830. Il fut ensuite nommé inspecteur de la cavalerie et siégea au comité de cette arme, le même qui rendit un avis positif sur le cheval d'Auzoux. Oudinot a laissé le souvenir d'un personnage compétent, réformant le casernement des chevaux pour accroître le niveau d'hygiène et souleva une grande polémique en proposant que l'administration des Haras soit intégrée au ministère de la Guerre. Les tensions diplomatiques de 1840 avaient entraîné l'achat de chevaux pour les remontes à l'étranger (les statistiques mentionnent 340000 chevaux, la majorité étant achetée en Allemagne). Il publia en 1842 Des remontes de l'armée, de leurs rapports avec l'administration des haras, un ouvrage qui déclencha de vives réactions, notamment des opposants à cette réforme destinée à répondre au manque cruel de chevaux de guerre.

36. - « Nous nous sommes bornés à reproduire, aussi fidèlement qu'il nous a été possible, des mâchoires naturelles prises dans notre collection et dans les collections de l'École vétérinaire d'Alfort; nous avons choisi les plus beaux types, et nous avons été aidé dans ce choix par M. le professeur Renault, directeur de cette école... ». Dans AUZOUX, Louis. Mâchoires du cheval et du bœuf. Paris : chez l'auteur, 1850.

37. - Narcisse Girard, 1796-1825, était le fils de Jean Girard, un professeur emblématique de l'École d'Alfort du début du XIX ${ }^{\mathrm{e}}$ siècle, qui en devint directeur. Narcisse devint lui-même vétérinaire ; diplômé d'Alfort en 1816, il fut nommé inspecteur vétérinaire des remontes à Caen en 1816 avant de devenir professeur à Alfort en 1821. Il mourut en 1825 alors qu'il achevait des études de médecine, emporté par les suites d'une piqûre anatomique qu'il s'était faite en pratiquant l'autopsie d'un des ses élèves. Il publia un long article consacré aux moyens de reconnaitre l'âge du cheval qu'il publia en 1824 dans le journal qu'il créa et qui devint par la suite le Recueil de Médecine Vétérinaire. L'Hippélikiologie fut publiée en 1828 par son père et connut deux rééditions.

38. - LECOQ, Félix. Traité de l'Extérieur du cheval. Paris : Vve Bouchard-Huzard, 1843.

39. - BRACY-CLARCK. Recherches sur la construction du sabot et suite d'expériences sur les effets de la ferrure. Paris: Vve Huzard, 1817. Le sabot était décomposé en muraille, sole, fourchette et périople.

40. - RIGOT était forcément bien au courant de la situation car Jacob était professeur de dessin à l'École d'Alfort et produisait notamment des dessins anatomiques dans cet établissement où Rigot était titulaire de la chaire.

41. - MINISTÈRE DE LA GUERRE. Cours d'équitation militaire à l'usage des corps de troupes à cheval. 2 volumes. Paris, Saumur : Anselin, Degouy, 1830. Ce cours avait été rédigé par Antoine Flandrin, 1798-18.., fils du célèbre professeur d'Alfort, Pierre Flandrin, un écuyer au caractère ombrageux, nommé professeur d'hippiatrique à l'École de Saumur et en 1822 à Saint-Cyr. Le second rédacteur était Jean-Baptiste Cordier, dit Fréville, 1771-1849, un écuyer de très grande classe qui fut nommé successivement à Saumur en 1815, à Saint-Cyr en 1822, à Versailles en 1824 et de nouveau à Saumur en 1825.

42. - Lettre du bureau de la remonte générale à Auzoux, en date du 21 juillet 1845. AN 242/API. 
43. - Lettre circulaire d'Auzoux aux commandants de régiments de cavalerie, 15 octobre 1845 . AN 242API/2.

44. - Lettre circulaire d'Auzoux aux commandants de régiments de cavalerie, 15 octobre 1845 . AN $242 \mathrm{API} / 2$.

45. - Lettre circulaire d'Auzoux aux colonels commandant les régiments, datée du 25 juillet 1847. AN 242API/2.

46. - AUZOUX, Louis. De l'utilité de l'anatomie clastique sous le rapport du choix, de l'emploi, de la conservation du cheval. Paris : l'auteur, 1847.

47. - JACQUEMIN, Maxime. Compte rendu de l'Anatomie clastique du Dr Auzoux et de l'influence qu'elle doit avoir sur l'Instruction de la Cavalerie. Paris : Firmin-Didot, 1847.

48. - AUZOUX, Louis. De l'utilité de l'anatomie clastique sous le rapport du choix, de l'emploi, de la conservation du cheval. Paris : l'auteur, 1847, p. 6.

49. - La remonte des armées consistait à fournir à une armée essentiellement hippomobile cavalerie, train d'artillerie, subsistances... - les chevaux nécessaires à leurs activités. Auzoux fut promu officier de la Légion d'honneur au titre des remontes.

50. - Son usine de Saint-Aubin-d'Éscroville était un modèle de ce point de vue. Les ouvriers y suivaient des cours d'anatomie, apprenaient à lire et écrire pour ceux qui étaient illettrés, pratiquaient le sport.

51. - RICHARD, Antoine. De la conformation du cheval selon les lois de la physiologie et de la mécanique. Paris : comptoir des imprimeurs-unis, 1847, p. 404.

52. - Ibid, p. 532.

53. - AUZOUX, Louis. Insuffisance, en France, du Cheval de Guerre et de luxe. Possibilité de l'obtenir en créant dans les Régiments de cavalerie des Écoles d'éleveurs au moyen du Cheval clastique du Docteur Auzoux. Paris : Firmin-Didot, 1854.

54. - AUZOUX, Louis. Leçons élémentaires d'anatomie et de physiologie humaine et comparée. Paris : Labé, 1858, p. 405.

55. - AUZOUX, Louis. De l'utilité de l'anatomie clastique sous le rapport du choix, de l'emploi, de la conservation du cheval. Paris : l'auteur, 1847, p. 9.

56. - AUZOUX, Louis. Des tares osseuses dans le Cheval. Paris : chez l'auteur, 1848.

57. - AUZOUX, Louis. Des Tares molles et osseuses dans le Cheval. Paris : chez l'auteur, 1853.

58. - AUZOUX, Louis. Mâchoires du cheval et du bœuf. Paris : chez l'auteur, 1850.

59. - Lettre de Jacquemin à Auzoux du 12 juillet 1845 : "Mon très cher et honoré maître, votre cheval est arrivé ici sans encombre à part toutefois une légère excoriation produite aux parties latérales les plus saillantes des fesses et des épaules par le frottement dont des coussinets ne garantissaient pas ces parties. Quoiqu'il en soit, votre précieux modèle a été accueilli avec enthousiasme, je commence à le démonter et à le remonter avec assez de facilité et nous allons en tirer bon parti». Le post-scriptum signalait: "Songez sérieusement aux tares et aux mâchoires pour la connaissance de l'âge. Vous en aurez un débit considérable et vous rendrez un immense service à la cavalerie ». AN 242API/2.

60. - Lettre de Jacquemin à Auzoux, datée du $1^{\mathrm{er}}$ octobre 1851. AN 242API/2.

61. - MINISTÈRE DE LA GUERRE. Traité pour la fourniture de 68 exemplaires du cheval artificiel. 12 juillet 1853. AN API242/3.

62. - Lettre du service de l'artillerie à Auzoux, datée du 21 février 1848, accusant réception du cheval clastique. AN API242/2.

63. - Mémoire manuscrit de Louis Auzoux en faveur de la création dans les départements d'un enseignement pour les éleveurs. Feuillet 10. AN 242API/2. Ce mémoire a été publié sous le titre : AUZOUX, Louis. Insuffisance des Chevaux forts et légers, du Cheval de Guerre et de luxe. Possibilité de l'obtenir en créant dans chaque département des Écoles d'éleveurs. Paris : Labé, 1860.

64. - Lettre circulaire du ministre de l'Agriculture aux préfets, datée du 3 août 1860. AN 242API/2. 
65. - Cette École, fondée par ordonnance royale du 24 octobre 1840, dispensait notamment des cours d'anatomie qui s'appuyaient sur le cheval clastique. Auzoux aurait été en droit d'espérer que chaque dépôt et haras en soit pourvu de la même façon puisqu'un enseignement avait été créé dans chaque institution, par arrêté ministériel du 7 juin 1837 ; on peut douter que cela ait été le cas.

66. - Lettre d'Armand Goubaux, directeur de l'École vétérinaire d'Alfort, à Louis Auzoux du 25 juin 1856: «Mon cher Docteur, Je vous adresse le cheval clastique ainsi que vous me le demandiez dans la lettre que vous m'avez fait remettre hier au soir. S'il vous était possible de remettre le nouveau modèle au commissionnaire de l'École. » AN 242API/2.

67. - Courrier de Félix Lecoq à Louis Auzoux en date du 10 janvier 1851: «J'ai l'honneur de vous prier de m'expédier le plus tôt qu'il vous sera possible, un exemplaire de mâchoires de cheval cotée sur votre catalogue au prix de deux cents francs. » AN 242API/2.

68. - Lettre de Félix Lecoq à Louis Auzoux en date du 20 janvier 1852 : «Mon cher Docteur, Je ne suis en arrière que d'un mois pour vous annoncer l'heureuse arrivée de tous vos clastiques dans notre école. Tout est venu à bon port ; le cheval seul a été un peu blessé par sa selle, qui a percé enveloppes et couverture et produit une légère dépression du pannicule charnu du côté gauche. $»$

69. - Les bons de commande de l'entreprise sont conservés aux Archives nationales. Leur analyse permettrait réellement de connaître l'ampleur de la diffusion internationale de ces pièces.

70. - Le Moniteur, 16 mars $1862, \mathrm{n}^{\circ} 75$.

71. - Lettre de Jacquemin à Auzoux, datée du 30 octobre 1848. AN 242API/2.

72. - Lettre du service des remontes à Auzoux, datée du 15 avril 1874. AN 242API/2.

73. - DUMONT, Barbara, DUPONT, Anne-Laurence, PAPILLON, Marie-Christine, JEANNEL, GaëlFrançois. "Technical study and conservation treatment of a horse model by Dr Auzoux". Studies in conservation, 2011, 56, p. 58-74.

74. - Certaines recevaient quinze couches de glacis.

75. - Il est situé 54 avenue de la Libération ; tel. 02-32-35-93-95.

\section{RÉSUMÉS}

Le docteur Louis Auzoux (1797-1880) est bien connu pour les mannequins anatomiques de papier qu'il produisit et exporta dans le monde entier. Si les modèles se rapportant à l'homme ont une force esthétique qui leur a donné une visibilité prépondérante, ils sont loin d'être les seuls que le célèbre industriel a conçus et commercialisés ; les animaux, les végétaux et tout particulièrement les fleurs, constituent une autre facette de son art. Mais les modèles dédiés au cheval eurent une importance toute particulière pour l'entreprise ; les chevaux de papier qu'il créa, les collections de tares et de mâchoires furent massivement achetées par l'administration de la Guerre qui en pourvut les régiments afin de former les recrues. Au travers de ces objets fascinants, c'est tout un programme d'amélioration de l'élevage du cheval qui fut ainsi mis en œuvre dans la France du milieu du XIX ${ }^{\mathrm{e}}$ siècle. Ces collections qui ont été diffusées dans le monde entier et qui ont fait l'orgueil de la France sont aujourd'hui abîmées, ignorées, dispersées et en grand danger de disparition.

The Louis Auzoux collections of anatomical horse models. Doctor Louis Auzoux (1797-1880) is well known for the anatomical models he made in papier mâché, models that were exported 
throughout the world. The models of the human body had aesthetic qualities that made them especially appreciated, but they are not the only pieces that the industrialists designed and sold. Animals, plants and flowers in particular demonstrate other aspects of his art. Models of horses were of special significance for the firm. Its papier maché horses and its collections of bone defects and horses' jaws were purchased in large numbers by the Ministry of War and used in regimental centres for training new recruits. These fascinating objects are a reflection of the programme that set out to improve horse-breeding in mid-nineteenth-century France. These collections which were diffused throughout the world and were a source of national pride are often badly damaged today, neglected and in danger of being lost.

INDEX

Mots-clés : Auzoux, modèle anatomique, papier mâché, cheval

Keywords : Auzoux, anatomical model, papier mâché, horse

\section{AUTEUR}

\section{CHRISTOPHE DEGUEURCE}

Professeur, Conservateur du musée Fragonard de l'École nationale vétérinaire d'Alfort, Centre de Recherche en Histoire Européenne Comparée, Université Paris-Est, École nationale vétérinaire d'Alfort cdegueurce@vet-alfort.fr 